\title{
Novel fast random search clustering algorithm for mixing matrix identification in MIMO linear blind inverse problems with sparse inputs
}

\author{
David Luengo , Sandra Monzón, Antonio Artés-Rodríguez \\ Department of Signal Processing and Communications, Universidad Carlos III de Madrid, 28911 Leganés (Madrid), Spain
}

\begin{abstract}
A B S T R A C T
In this paper we propose a novel fast random search clustering (RSC) algorithm for mixing matrix identification in multiple input multiple output (MIMO) linear blind inverse problems with sparse inputs. The proposed approach is based on the clustering of the observations around the directions given by the columns of the mixing matrix that occurs typically for sparse inputs. Exploiting this fact, the RSC algorithm proceeds by parameterizing the mixing matrix using hyperspherical coordinates, randomly selecting candidate basis vectors (i.e. clustering directions) from the observations, and accepting or rejecting them according to a binary hypothesis test based on the Neyman-Pearson criterion. The RSC algorithm is not tailored to any specific distribution for the sources, can deal with an arbitrary number of inputs and outputs (thus solving the difficult under-determined problem), and is applicable to both instantaneous and convolutive mixtures. Extensive simulations for synthetic and real data with different number of inputs and outputs, data size, sparsity factors of the inputs and signal to noise ratios confirm the good performance of the proposed approach under moderate/high signal to noise ratios.
\end{abstract}

\section{Introduction}

Multiple input multiple output (MIMO) linear blind inverse problems are concerned with the estimation of a set of input signals (often called sources) from a collection of output signals (also called observations or measurements) when the inputoutput relationship (mixture) is linear but unknown. Blind inverse problems include blind source separation (BSS) and independent component analysis (ICA) [1-3], blind deconvolution (BDE) $[4,5]$, blind channel/system identification (BID) and blind equalization (BEQ) [6,7]. These problems can be extremely difficult to solve, since the only information available is typically some broad statistical assumption about the inputs (e.g. their independence), a limited number of observations, and a general knowledge about the mixing process followed to obtain them (e.g. its linearity and whether the mixture is instantaneous or convolutive). Using this information the inversion algorithm must be able to estimate the number of inputs (which may be unknown) and the system's memory (for the convolutive case), identify the mixing matrix (either implicitly or explicitly), and extract the inputs. In this paper we focus on the mixing matrix identification stage.

On the one hand, many excellent signal processing and machine learning methods have been developed for solving this problem over the last two decades [3]: information maximization techniques, algorithms based on higher order statistics, subspace methods, maximum likelihood (ML) and maximum a posteriori (MAP) approaches, etc. However, most of these techniques have been developed considering a particular probabilistic model for the inputs (e.g. Bernoulli-Gaussian or Laplacian models), mixture model (e.g. instantaneous or convolutive) and/or application (e.g. BSS or BDE), and their extension to different settings is often not straightforward. Besides, they usually cannot deal with the difficult under-determined case (i.e. more inputs than outputs), their computational cost is frequently excessive for practical applications (where a large number of samples may be available) and some of them lack robustness for application under noisy conditions.

On the other hand, several algorithms that can deal with the under-determined case have been developed for sparse input signals. Lee et al. proposed an approximate maximum likelihood approach with good performance, but high computational cost for some practical applications [8]. Hence, several fast and simple techniques based on the clustering of the observations around the 
directions given by the columns of the mixing matrix (also called line orientation clustering methods) have been proposed. Bofill and Zibulevsky developed a potential function approach working in the angular domain which is fast, simple and effective in high SNR situations [9]. Alternative approaches in the angular domain using a Parzen window density estimator [10] or histogram-based techniques [11] have also been proposed. Unfortunately, all of these techniques were initially designed for only two outputs and are difficult to extend to more general mixtures. Other geometric methods based on the projection of the data onto the surface of a unit sphere [12,13] or modified $k$-means approaches [14-16] allow working with an arbitrary number of observations, but their performance is very sensitive to noise. Recently, more sophisticated approaches based on the E-M algorithm [17], eigenvalue or singular value decomposition techniques $[18,19]$ and spectral clustering [20] have also been proposed. Once more, these methods provide a good performance at the expense of a high computational cost.

Therefore, even though many clustering algorithms have been proposed for the estimation of the mixing matrix for sparse inputs, there is still a need for simple, effective and robust techniques that can deal with a potentially large number of outputs. In particular, clustering in the angular domain, where noise may be reduced due to the compressive nature of the arctan function required for angle calculation, has not been fully exploited yet. Most of the angular clustering techniques proposed so far [9-11] focus on the two outputs case, and cannot be easily extended to a higher number of outputs. The only angular clustering approach theoretically valid for an arbitrary number of outputs is the one proposed in [21], which is based on hyperspherical coordinates and one-dimensional projections of the higher dimensional angular vector. Unfortunately, the exponential increase in complexity with the dimension of the problem limits its applicability to a reduced number of inputs and outputs (e.g. three outputs and four inputs [21]).

In this paper we propose a novel fast clustering algorithm in the angular domain based on the use of hyperspherical coordinates as in [21]. However, instead of the complicated projections system implemented there, we propose to use a simple random search method for selecting candidate basis vectors (i.e. clustering directions) from the observations, accepting or rejecting them according to a binary hypothesis test based on the NeymanPearson criterion [22]. This algorithm, called random search clustering (RSC), is not tailored to any specific distribution for the sources, can deal with an arbitrary number of inputs and outputs (thus solving the difficult under-determined problem) with a linear increase in complexity, and is applicable to both instantaneous and convolutive mixtures. Besides, it allows us to extend the complete inversion strategy described in [23] to an arbitrary number of outputs.

The paper is structured as follows. In Section 2 we describe the mathematical model assumed for the mixture, as well as the general probabilistic model used for the inputs, which emphasizes their sparsity. Then, Section 3 provides an overview of the complete inversion strategy, enumerating the three main steps involved and focusing on the two outputs case, where a simple histogram-based approach can be used to estimate the mixing matrix. This is followed by a detailed description of the novel random search clustering algorithm proposed for estimating the columns of the mixing matrix in Section 4 , whereas Section 5 concentrates on the parameter selection procedure. Simulation results are presented in Section 6 for synthetic and real data with different number of inputs and outputs, data size, sparsity factors of the inputs and signal to noise ratios. Finally, the conclusions and future lines close the paper in Section 7.

\section{Problem statement and mathematical model}

\subsection{Input-output relationship: linear convolutive mixture}

Let us assume that we have $Q>1$ correlated outputs or observations, $y_{q}(t), R \geq 1$ independent inputs or sources, $f_{r}(t)$, and an input-output relationship given by a linear convolutive mixture,

$y_{q}(t)=\sum_{r=1}^{R} h_{q r}(t) * f_{r}(t)+w_{q}(t)$

where $w_{q}(t)$ represents the additive noise associated to the $q$-th output, $h_{q r}(t)$ denotes the impulse response of the linear timeinvariant (LTI) channel between the $r$-th input and the $q$-th output, and $*$ indicates the standard linear convolution operator. In a practical situation we usually have only $N$ samples per channel available, taken at regular intervals with a sampling frequency $f_{s}=1 / T_{s}$. In this case, the commonly used discretetime equivalent model of $(1)$ is $[23,47,48]$

$y_{q}[n]=\sum_{r=1}^{R} \sum_{m=0}^{L_{q r}-1} h_{q r}[m] f_{r}[n-m]+w_{q}[n]$,

where $0 \leq n \leq N-1, h_{q r}[m]=h_{q r}\left(m T_{s}\right)$ for $0 \leq m \leq L_{q r}-1$ is the $(q, r)$ th discrete-time equivalent channel, with effective length $L_{q r}$, obtained by uniformly sampling the $(q, r)$-th continuous-time channel, $h_{q r}(t)$, and $f_{r}[n-m]=f_{r}\left((n-m) T_{s}\right)$ and $w_{q}[n]=w_{q}\left(n T_{s}\right)$ are, respectively, the discretized sources and noise, obtained in an identical way.

Mathematically, all the information available in this discretetime model can be expressed compactly in matrix form as

$\mathbf{Y}=\mathbf{H S}+\mathbf{W}=\mathbf{X}+\mathbf{W}$,

where $\mathbf{Y}=[\mathbf{y}[0], \ldots, \mathbf{y}[N-1]]$ is the $Q \times N$ noisy output matrix, constructed stacking $N$ consecutive output vectors, $\mathbf{y}[n]=$ $\left[y_{1}[n], \ldots, y_{Q}[n]\right]^{T}$;

$\mathbf{H}=\left[\begin{array}{ccc}\mathbf{h}_{11}^{\top} & \cdots & \mathbf{h}_{1 R}^{\top} \\ \vdots & & \vdots \\ \mathbf{h}_{Q 1}^{\top} & \cdots & \mathbf{h}_{\mathrm{QR}}^{\top}\end{array}\right]$,

with $\mathbf{h}_{q r}^{\top}=\left[h_{q r}[0], \ldots, h_{q r}\left[L_{r}-1\right]\right]$ and $L_{r}=\max _{q} L_{q r}$, is the $Q \times L$ mixing matrix $\left(L=\sum_{r=1}^{R} L_{r}\right)$, which contains the relevant samples from all the input-output discrete-time equivalent channels; $\mathbf{S}=[\mathbf{s}[0], \ldots$, $\mathbf{s}[N-1]]^{T}$, with $\mathbf{s}[n]=\left[\mathbf{s}_{1}^{\top}[n], \ldots, \mathbf{s}_{R}^{\top}[n]\right]^{\top}$ and $\mathbf{s}_{r}^{\top}[n]=\left[f_{r}[n], f_{r}[n-1]\right.$, . . $\left.f_{r}\left[n-\left(L_{r}-1\right)\right]\right]$, is the $L \times N$ input matrix, which contains $N$ consecutive input vectors; $\mathbf{W}=[\mathbf{w}[0], \ldots, \mathbf{w}[N-1]]$ is the $Q \times N$ noise matrix, with $\mathbf{w}[n]=\left[w_{1}[n], \ldots, w_{Q}[n]\right]^{T}$ and $w_{q}[n]$ independent zero-mean Gaussian random variables with variance $\sigma_{w_{g}}^{2}$, i.e. $\mathbf{w}[n] \sim \mathcal{N}\left(\mathbf{0}, \Sigma_{\mathbf{w}}\right) \quad$ with $\quad \boldsymbol{\Sigma}_{\mathbf{w}}=\operatorname{diag}\left(\sigma_{w_{1}}^{2}, \ldots, \sigma_{w_{Q}}^{2}\right) ; \quad$ and, finally, $\mathbf{X}=\mathbf{H S}=[\mathbf{x}[0], \ldots, \mathbf{x}[N-1]]$ is the $Q \times N$ noiseless output matrix, with $\mathbf{x}[n]=\left[x_{1}[n], \ldots, x_{Q}[n]\right]^{T}$.

Note that, although the original number of inputs in the problem is $R$, the mixture model in matrix form has $L \geq R$ inputs. These additional inputs simply correspond to the last $L_{r}-1$ samples from each original input: $f_{r}[n-1], \ldots, f_{r}\left[n-\left(L_{r}-1\right)\right]$ for $1 \leq r \leq R$. As a result, the system described by (3) becomes under-determined whenever $L>Q$, even if the original number of inputs is lower than the number of outputs (i.e. $R<Q$ ). Moreover, the structure of both $\mathbf{H}$ and $\mathbf{s}[n]$ depends on the mixture's memory, i.e. on whether it is instantaneous or convolutive and on the length of each subchannel for this last case. However, regardless of its precise structure, the mixing matrix can always be described as $\mathbf{H}=\left[\mathbf{h}_{1}, \ldots, \mathbf{h}_{L}\right]^{T}$, where $\mathbf{h}_{\ell}=\left[h_{\ell}(1), \ldots, h_{\ell}(\mathbf{Q})\right]^{\top}$ denotes the $\ell$-th column of $\mathbf{H}$. Similarly, the input vector can be expressed as $\mathbf{s}[n]=\left[s_{1}[n], \ldots, s_{L}[n]\right]^{T}$, with $s_{\ell}[n]$ denoting the $\ell$-th input to the mixture at the $n$-th sampling instant, 
which may actually be a delayed sample of the $r$-th original input for $1 \leq r \leq \ell$.

In the sequel we exploit this formulation, since it is wellknown that a matrix-vector multiplication can be expressed as a linear combination of the columns of the matrix with weights given by the components of the vector [24,9]. Hence, the matrixvector multiplication required to obtain the noiseless outputs at each time instant, $\mathbf{x}[n]=\mathbf{H s}[n]$, becomes a linear combination of the columns of $\mathbf{H}, \mathbf{h}_{\ell}$, with weights given by the corresponding elements of the input vector, $s_{\ell}[n]$. Similarly, the $n$-th sample of the noisy outputs is given by this linear combination plus the noise vector:

$\mathbf{y}[n]=\mathbf{x}[n]+\mathbf{w}[n]=\mathbf{H s}[n]+\mathbf{w}[n]=\sum_{\ell=1}^{L} s_{\ell}[n] \mathbf{h}_{\ell}+\mathbf{w}[n]$.

Therefore, the columns of the mixing matrix, $\mathbf{h}_{\ell}$, form a basis in the $Q$-dimensional measurement space, ${ }^{1}$ and (5) can be seen as an expansion of $\mathbf{y}[n]$ in terms of this set of non-orthogonal basis vectors, with $s_{\ell}[n]$ being the coefficients of the basis expansion. Thus, identifying $\mathbf{H}$ is equivalent to inferring the optimum set of basis vectors, $\mathbf{h}_{\ell}(1 \leq \ell \leq L)$, and inverting the mixture amounts to estimating the coefficients of the basis expansion, $s_{\ell}[n]$ $(1 \leq \ell \leq L, 0 \leq n \leq N-1)$.

\subsection{General probabilistic model: white, independent and sparse inputs}

Considering white and independent sources, as in the standard ICA model [1], the joint probability density function (PDF) for all the samples from all the inputs in (2) is given by

$p_{\mathbf{F}}(\mathbf{f})=\prod_{r=1}^{R} \prod_{n=0}^{N-1} p_{F_{r}}\left(f_{r}[n]\right)$,

where $p_{F_{r}}\left(f_{r}[n]\right)$ is the PDF associated to the $n$-th sample of the $r$-th source. For this PDF we follow a similar approach to [23], where the sparsity of the inputs is emphasized explicitly by formulating $p_{F_{r}}\left(f_{r}[n]\right)$ as the mixture of two distributions: a first term corresponding to $f_{r}[n]$ being inactive (i.e. $f_{r}[n]=0$ ) and a second term describing the distribution of the active samples (i.e. $f_{r}[n] \neq 0$ ). Mathematically, defining an indicator function

$\|\left(f_{r}[n]\right)=\mathbb{\Xi}_{r}[n]= \begin{cases}1, & f_{r}[n] \neq 0, \\ 0, & f_{r}[n]=0,\end{cases}$

we may express the desired PDF as

$$
\begin{aligned}
p_{F_{r}}\left(f_{r}[n]\right) & =\eta_{r} p\left(f_{r}[n] \mid \square_{r}[n]=0\right)+\left(1-\eta_{r}\right) p\left(f_{r}[n] \mid \nabla_{r}[n]=1\right) \\
& =\eta_{r} \delta\left(f_{r}[n]\right)+\left(1-\eta_{r}\right) p_{F_{r}}^{(\mathcal{A})}\left(f_{r}[n]\right),
\end{aligned}
$$

where $0 \leq \eta_{r} \leq 1$ is the sparsity factor for the $r$-th input, which indicates its probability of being inactive (i.e. $\eta_{r}=\operatorname{Pr}\left\{f_{r}[n]=0\right\}$ ), $p\left(f_{r}[n] \mid \|_{r}[n]=0\right)=\delta\left(f_{r}[n]\right)$, with $\delta(x)$ denoting Dirac's delta, is the inactive PDF associated to $f_{r}[n]$, and $p_{F_{r}}^{(\mathcal{A})}\left(f_{r}[n]\right)=p\left(f_{r}[n] \mid \square_{r}[n]=1\right)$ denotes its active PDF.

The last element required is the active PDF of the sources. Although the proposed algorithm does not make use of this PDF, in the sequel we describe three common PDFs used to generate the synthetic data for the simulations. As a first example, we consider a zero-mean normal distribution with variance $\sigma_{f}^{2}$ for all the inputs,

$p_{F_{r}}^{(\mathcal{A})}\left(f_{r}[n]\right)=\mathcal{N}\left(f_{r}[n] \mid 0, \sigma_{f}^{2}\right)=\frac{1}{\sqrt{2 \pi \sigma_{f}^{2}}} \exp \left(-\frac{f_{r}^{2}[n]}{2 \sigma_{f}^{2}}\right)$.

${ }^{1}$ For $L>Q$ (underdetermined case) the columns of $\mathbf{H}$ form an overcomplete basis [25].
Introducing this PDF in (8) we obtain the well-known BernoulliGaussian (B-G) model [26],

$p_{F_{r}}\left(f_{r}[n]\right)=\eta_{r} \delta\left(f_{r}[n]\right)+\frac{1-\eta_{r}}{\sqrt{2 \pi \sigma_{f}^{2}}} \exp \left(-\frac{f_{r}^{2}[n]}{2 \sigma_{f}^{2}}\right)$,

extensively used as a prior for sparse inputs in many applications such as seismic deconvolution [27,28], non-destructive evaluation [29], optical coherence tomography [30], multipath channel estimation [31] and impulsive noise modelling [32,33] in digital communications, or spectral analysis of astrophysical data [34] Fig. 1 illustrates the spiky nature of signals generated by the $B-G$ model by displaying $N=1000$ samples of the $Q=3$ outputs, obtained for an instantaneous noiseless mixture with $L=R=5$ white and independent inputs, distributed according to (10) with a common sparsity factor $\eta=0.75$, and the mixing matrix obtained from (65). Note that the largest variance of $y_{3}[n]$ is simply due to the fact that the squared norm of the third row of $\mathbf{H}$ is approximately 4 , whereas the squared norm of the other two rows is around 0.4 and 0.5 respectively.

As a second example we consider another widely used probabilistic model for sparse applications, the Laplacian PDF with zero mean and variance $\sigma_{f}^{2}$ :

$p_{F_{r}}^{(\mathcal{A})}\left(f_{r}[n]\right)=\mathcal{L}\left(f_{r}[n] \mid 0, \sigma_{f}\right)=\frac{1}{\sqrt{2 \sigma_{f}^{2}}} \exp \left(-\frac{\sqrt{2}\left|f_{r}[n]\right|}{\sigma_{f}}\right)$

Speech samples during voice activity intervals are well described (both in the time and frequency domains) by a Laplacian PDF [35]. Hence, this PDF has been proposed as prior for applications such as voice activity detection [36] or speech enhancement [37]. The Laplacian PDF has also been widely used as a prior for modelling sparse signals since it was first proposed by Alliney and Ruzinsky [38] and Tibshirani [39]. However, in spite of the fact that a Laplacian prior promotes the sparsity of the outputs, the Laplacian PDF is not a sparse distribution itself [40]. Combining a delta function located at zero and a Laplacian PDF we obtain the Bernoulli-Laplacian (B-L) model,

$p_{F_{r}}\left(f_{r}[n]\right)=\eta_{r} \delta\left(f_{r}[n]\right)+\frac{1-\eta_{r}}{\sqrt{2 \sigma_{f}^{2}}} \exp \left(-\frac{\sqrt{2}\left|f_{r}[n]\right|}{\sigma_{f}}\right)$.
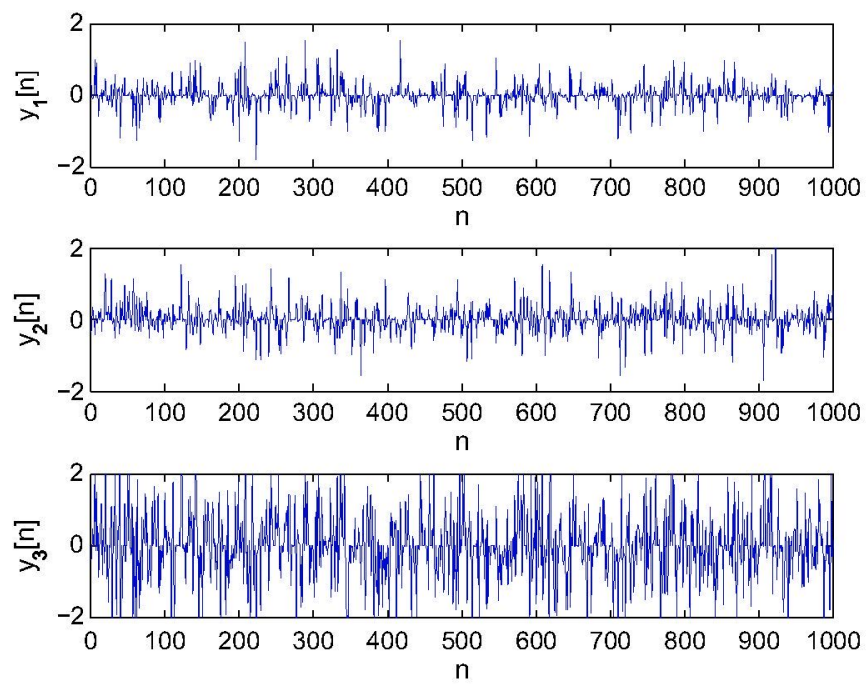

Fig. 1. $N=1000$ samples of the $Q=3$ outputs of an instantaneous noiseless mixture with $L=R=5$ inputs generated using the $\mathrm{B}-\mathrm{G}$ model, $\eta=0.75$ and $\mathbf{H}$ given by (65). 
This idea was proposed originally by Johnstone and Silverman [41], and applied by Ting et al. to the reconstruction of sparse images [40].

Finally, as a third example we consider a uniform distribution with zero mean and variance $\sigma_{f}^{2}$ for all the inputs,

$p_{F_{r}}^{(\mathcal{A})}\left(f_{r}[n]\right)=\frac{1}{2 \sqrt{3 \sigma_{f}^{2}}}\left[u\left(f_{r}[n]+\sqrt{3 \sigma_{f}^{2}}\right)-u\left(f_{r}[n]-\sqrt{3 \sigma_{f}^{2}}\right)\right]$,

where $u(x)$ denotes Heaviside's unit step function. Substituting (13) in (8) we obtain the Bernoulli-Uniform (B-U) model,

$p_{F_{r}}\left(f_{r}[n]\right)=\eta_{r} \delta\left(f_{r}[n]\right)+\frac{1-\eta_{r}}{2 \sqrt{3 \sigma_{f}^{2}}}\left[u\left(f_{r}[n]+\sqrt{3 \sigma_{f}^{2}}\right)-u\left(f_{r}[n]-\sqrt{3 \sigma_{f}^{2}}\right)\right]$,

used in [42] for the evaluation of an ICA algorithm. The B-U model does not have any straightforward physical interpretation and has not been used for any practical application as far as we know. However, the uniform distribution is a well-known example of platykurtic or sub-Gaussian distribution (as opposed to the Laplacian PDF, which is leptokurtic or super-Gaussian) and allows us to check the performance of our method in a situation where the active samples are concentrated around the origin.

\section{General problem solution for sparse inputs}

3.1. Global method: source detection, mixing matrix identification and mixture inversion

In general, solving any linear blind inverse problem requires three broad steps: inferring the number of inputs present in the mixture, altogether with the mixture's memory (i.e. the values of $R$ and $L_{q r}$ for $1 \leq q \leq Q, 1 \leq r \leq R$ ); identifying the mixing matrix; and, finally, inverting the mixture and recovering the inputs. A general algorithm for MIMO linear blind inverse problems which solves these three stages by explicitly exploiting the sparsity of the inputs was described in [23]. Recalling (5) we notice that, when only the $\ell$-th input $(1 \leq \ell \leq L)$ is active (i.e. $s_{\ell}[n] \neq 0$ and $s_{m}[n]=0$ for $1 \leq m \leq L$ with $m \neq \ell$ ), the output is given by

$\mathbf{y}_{\ell}[n]=\mathbf{x}_{\ell}[n]+\mathbf{w}[n]=s_{\ell}[n] \mathbf{h}_{\ell}+\mathbf{w}[n]$.

Hence, in the absence of noise, the sparsity of the inputs causes the Q-dimensional output vectors to be frequently colinear with the columns of $\mathbf{H}$, i.e. to belong to one of the $L$ one-dimensional subspaces spanned by $\mathbf{h}_{\ell}$ for $1 \leq \ell \leq L$. This situation can be clearly appreciated in a scatter plot which, for the two outputs case, plots $y_{2}[n]$ versus $y_{1}[n]$ [23]. The scatter plot can still be displayed in the three outputs case, as shown in Fig. 2, which represents the three-dimensional scatter plot corresponding to $N=2000$ samples of the $Q=3$ noiseless observations $\left(y_{1}[n], y_{2}[n]\right.$ and $\left.y_{3}[n]\right)$ of an instantaneous mixture obtained using $L=R=5$ white and independent inputs distributed according to the B-G model, a common sparsity factor $\eta=0.7$ and a mixing matrix constructed using (65). On the one hand, Fig. 2(a) shows the full scatter plot using all the observations, where the five onedimensional subspaces spanned by $\mathbf{h}_{1}, \ldots, \mathbf{h}_{5}$ cannot be clearly distinguished due to the presence of many "outliers" (i.e. samples not belonging to any of those subspaces). On the other hand, Fig. 2(b) shows the partial scatter plot using only samples colinear with columns of $\mathbf{H}$, demonstrating that the outputs tend to cluster around the directions of the mixing matrix.

For $Q>3$ outputs the global $(Q-1)$-dimensional scatter plot cannot be displayed. However, the sparsity of the outputs and their clustering around the directions spanned by the columns of $\mathbf{H}$ can still be seen by taking bidimensional projections of the
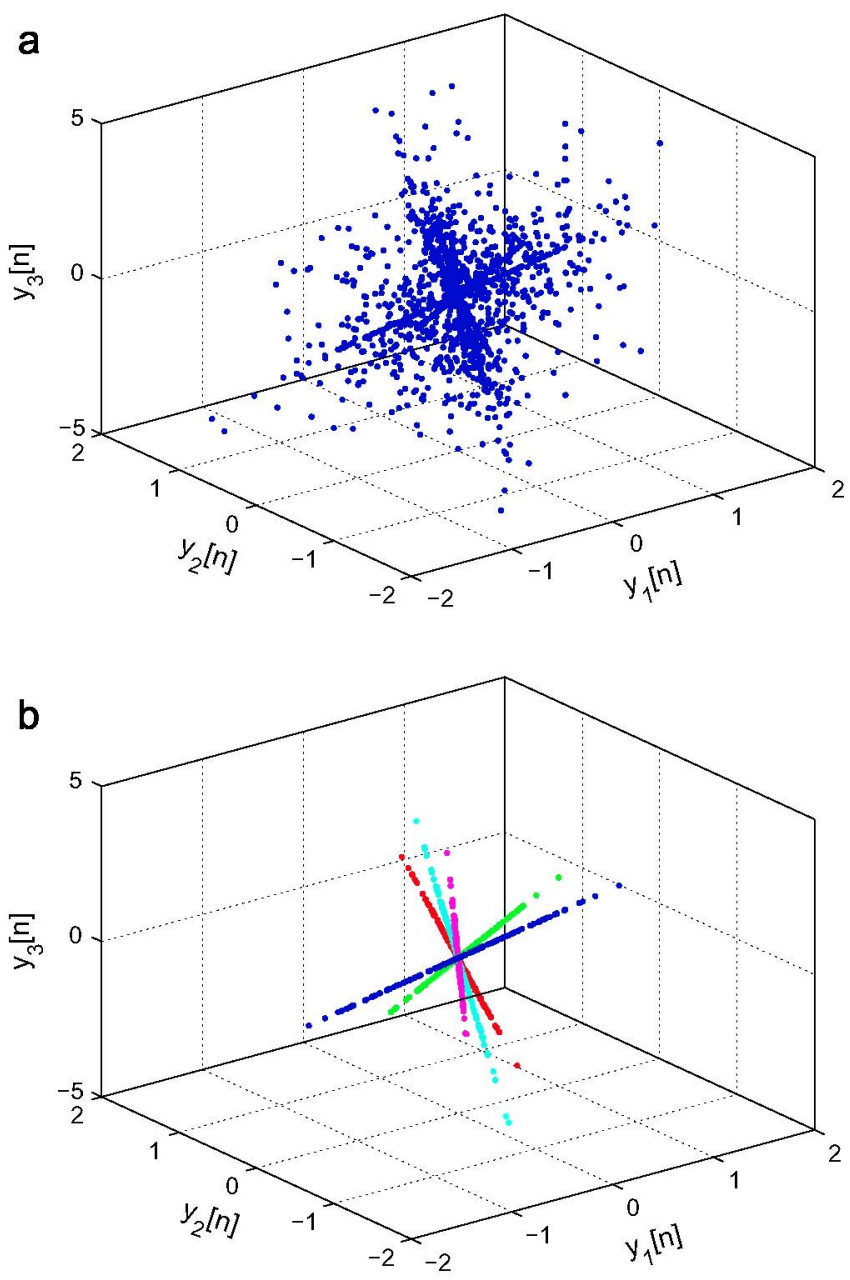

Fig. 2. Noiseless three-dimensional scatter plots for $Q=3$ outputs, $L=5$ inputs, mixing matrix constructed using (65), $N=2000$ samples and sparsity factor $\eta=0.7$. (a) Full scatter plot using all observations. (b) Partial scatter plot using only samples colinear with columns of $\mathbf{H}$.

outputs. This is illustrated in Fig. 3, which shows four twodimensional scatter plots (out of the $Q(Q-1) / 2=10$ possible), obtained in this case by plotting $y_{q}[n](2 \leq q \leq Q=5)$ versus $y_{1}[n]$. In these scatter plots each colour represents samples colinear with one of the basis vectors, whereas black dots indicate samples not colinear with any column of $\mathbf{H}$. Note how angles that cannot be distinguished at all in some of the plots are clearly separated in some other cases.

Under low/moderate noise conditions the outputs remain approximately colinear with the columns of $\mathbf{H}$, and, as a result, they tend to cluster around the one-dimensional subspaces described before. Therefore, estimating $\mathbf{H}$ still amounts to inferring these one-dimensional subspaces, which agree with the directions of maximum data density and can be estimated using line orientation clustering techniques.

\subsection{Histogram-based clustering for two outputs}

For $Q=2$ a simple histogram-based clustering technique was described in $[11,23]$ to estimate the columns of the mixing matrix. This method is based on a novel parameterization of $\mathbf{H}$ using polar coordinates for its columns, which can be formulated compactly as the product of two matrices,

$\mathbf{H}=\boldsymbol{\Phi} \boldsymbol{\Lambda}$ 


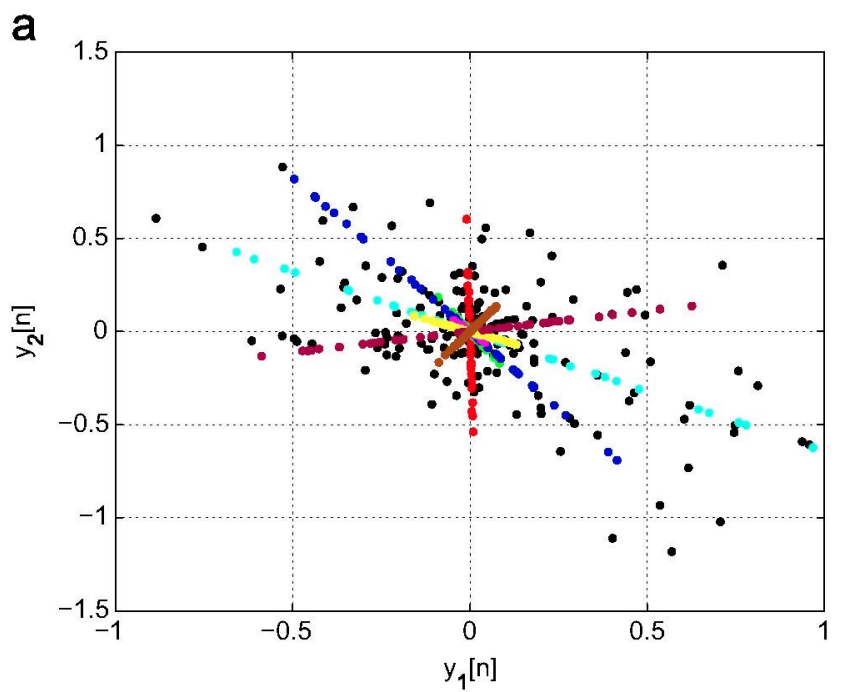

b
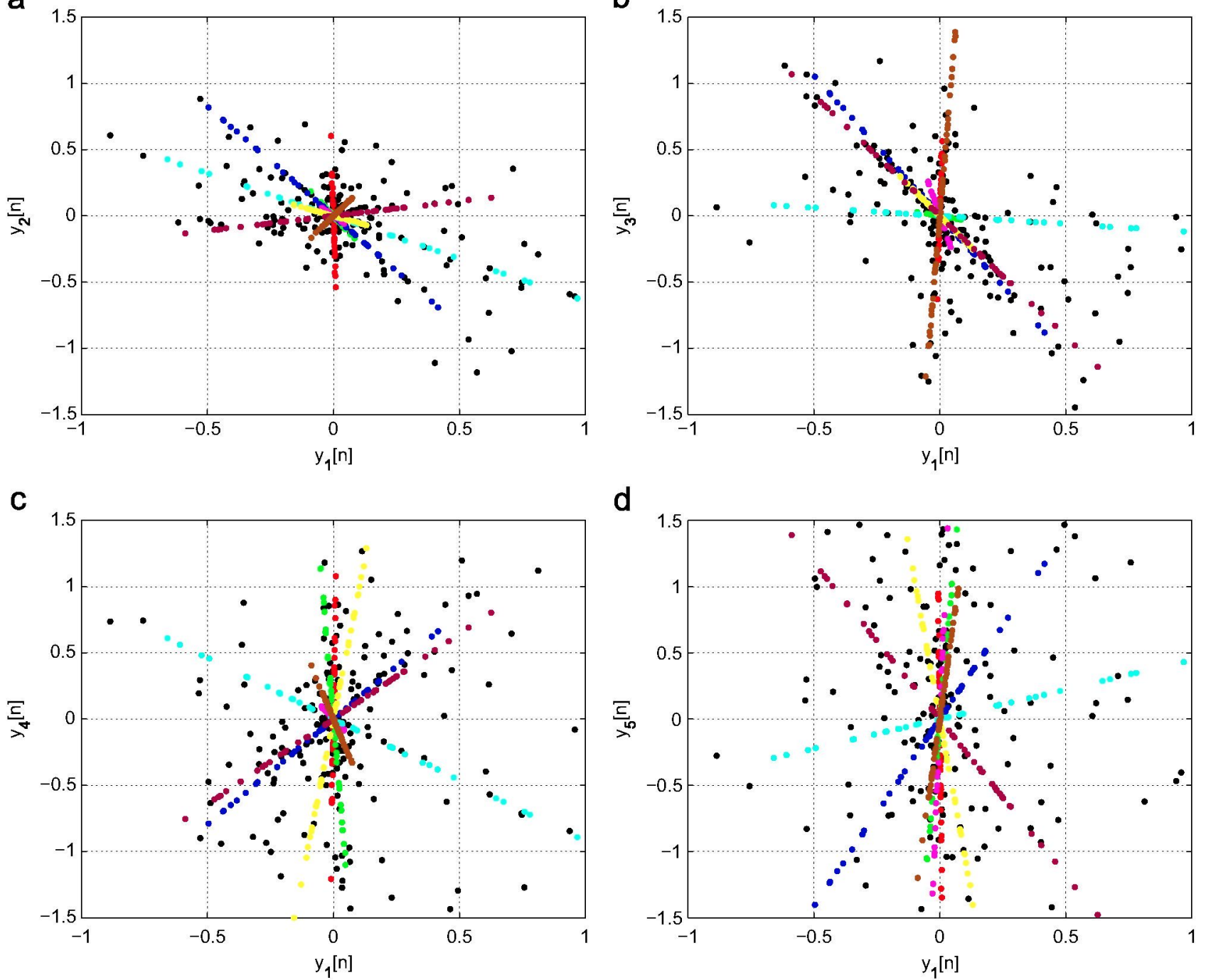

d

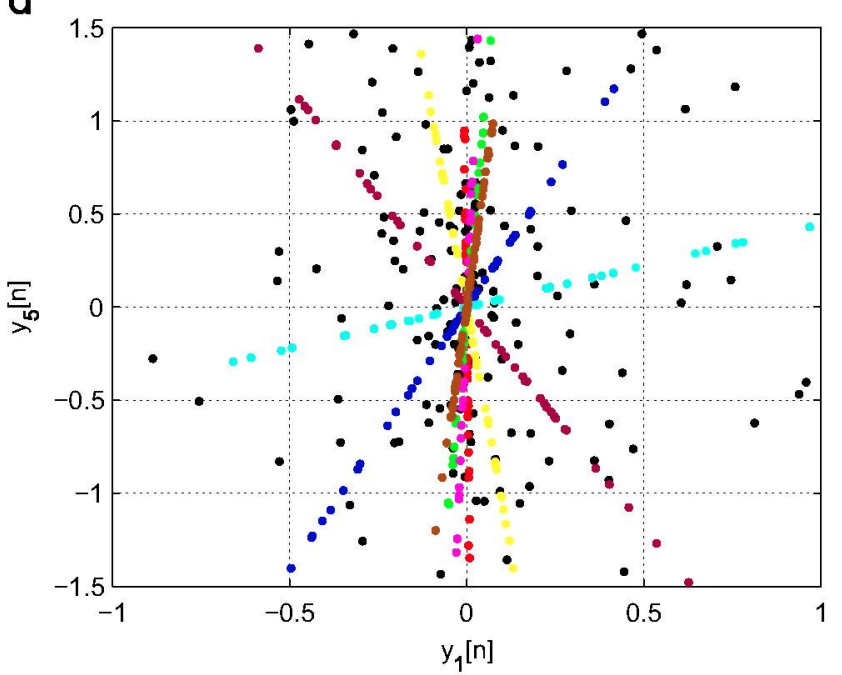

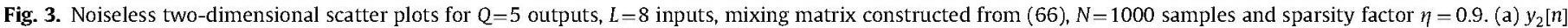
vs. $y_{1}[n]$. (b) $y_{3}[n]$ vs. $y_{1}[n]$. (c) $y_{4}[n]$ vs. $y_{1}[n]$. (d) $y_{5}[n]$ vs. $y_{1}[n]$.

where $\Lambda$ is an $L \times L$ diagonal norm matrix,

$\boldsymbol{\Lambda}=\operatorname{diag}(\lambda)=\operatorname{diag}\left(\left\|\mathbf{h}_{1}\right\|, \ldots,\left\|\mathbf{h}_{L}\right\|\right)$,

with $\lambda=\left[\left\|\mathbf{h}_{1}\right\|, \ldots,\left\|\mathbf{h}_{L}\right\|^{\top}\right.$ and $\left\|\mathbf{h}_{\ell}\right\|$ the $L_{2}$ norm of the $\ell$-th column of $\mathbf{H}$,

$\left\|\mathbf{h}_{\ell}\right\|=\left(\mathbf{h}_{\ell}^{\top} \mathbf{h}_{\ell}\right)^{1 / 2}=\left[h_{\ell}^{2}(1)+h_{\ell}^{2}(2)\right]^{1 / 2}$,

and $\boldsymbol{\Phi}$ is a $2 \times L$ angular matrix,

$\boldsymbol{\Phi}=\left[\begin{array}{c}\cos (\boldsymbol{\theta}) \\ \sin (\boldsymbol{\theta})\end{array}\right]=\left[\begin{array}{ccc}\cos \left(\theta_{1}\right) & \cdots & \cos \left(\theta_{L}\right) \\ \sin \left(\theta_{1}\right) & \cdots & \sin \left(\theta_{L}\right)\end{array}\right]$,

with $\theta=\left[\theta_{1}, \ldots, \theta_{L}\right]^{\top}$ and $-\pi \leq \theta_{\ell}<\pi$ the four quadrant angle of the $\ell$-th column of $\mathbf{H}$, obtained from the two quadrant version,

$\tilde{\theta}_{\ell}=\arctan \left(\frac{h_{\ell}(2)}{h_{\ell}(1)}\right)$

$-\pi / 2 \leq \tilde{\theta}_{\ell}<\pi / 2$, adding or subtracting $\pi$ radians when $h_{\ell}(1)<0$,

$\theta_{\ell}= \begin{cases}\tilde{\theta}_{\ell}, & h_{\ell}(1)>0, \\ \tilde{\theta}_{\ell}+\pi, & h_{\ell}(1)<0, h_{\ell}(2)>0, \\ \tilde{\theta}_{\ell}-\pi, & h_{\ell}(1)<0, h_{\ell}(2)<0 .\end{cases}$
Table 1

Summary of the general inversion approach for linear MIMO blind inverse problems described in [23].

1. Detecting the number of inputs present in the mixture (i.e. inferring the values of $R$ and $L_{\tau}$ ) using information theoretic criteria

2. Estimating the mixing matrix:

(a) Finding the angular vector $\theta$ (i.e. estimating the directions of the $L$ clusters) using a histogram-based approach and selecting the $L$ highest bins, as described in Section 3.2

(b) Inferring the norm vector $\lambda$ (i.e. estimating the dispersion of the $L$ clusters) applying a moment-based estimator to the outputs belonging to each of the selected bins

(c) Sorting the columns of the mixing matrix, to avoid permutation ambiguities, by exploiting the temporal correlation of the inputs

3. Inverting the mixture using the pseudoinverse as the canonical solution with minimum $L_{2}$ norm

Taking advantage of this parameterization, the algorithm solves the three problems associated to inferring the mixing matrix (finding $\theta_{\ell}$, estimating $\left\|\mathbf{h}_{\ell}\right\|$ and sorting the columns) sequentially. Table 1 summarizes the three main parts of the algorithm, emphasizing the mixing matrix identification step, which includes the key stage of this approach: locating each of 
Table 2

Histogram-based algorithm for identifying clustering directions when $Q=2$ $[11,23]$.

1. Construct a histogram with $N_{b}$ bins in the range $[0, \pi)$ (i.e. bin size $\Delta \theta=\pi / N_{b}$, with $k$-th bin, $1 \leq k \leq N_{b}$, centred around $\left.\pi(k-1 / 2) / N_{b}\right)$, using the angles estimated from the observations as

$$
\hat{\theta}[n]=\arctan \left(\frac{y_{2}[n]}{y_{1}[n]}\right)
$$

2. For $\ell=1, \ldots, L$ :

(a) Find the largest bin in the histogram, $k_{\ell}\left(1 \leq k_{\ell} \leq N_{b}\right)$, and its central position, $\bar{\theta}_{\ell}=\pi\left(k_{\ell}-1 / 2\right) / N_{b}$

(b) Select all the angles from the observations falling inside the selected bin (i.e. those within $\Delta \theta / 2$ angular distance of $\bar{\theta}_{\ell}$ ), constructing the index set for the $\ell$-th angle,

$$
\Pi_{\ell}=\left\{n:\left|\hat{\theta}[n]-\bar{\theta}_{\ell}\right| \leq \Delta \theta / 2\right\}
$$

(c) Estimate the $\ell$-th angle as an average of the angles associated to all the elements in $\Pi_{\ell}$ :

$$
\hat{\theta}_{\ell}=\frac{1}{\left|\Pi_{\ell}\right|} \sum_{n \in \Pi_{\ell}} \hat{\theta}[n]
$$

where $\left|\Pi_{\ell}\right|$ denotes the cardinality of $\Pi_{\ell}$.

(d) Set to zero all the bins from the $\left(k_{\ell}-N_{z}\right)$-th to the $\left(k_{\ell}+N_{z}\right)$-th, effectively creating an angular exclusion set composed of all the angles within angular distance $\pi\left(N_{z}+1 / 2\right) / N_{b}$ radians of $\bar{\theta}_{\ell}$, to avoid double counting of clusters associated to the same angle

the $L$ clusters. For $Q=2$ this is equivalent to estimating the angle of each column of $\mathbf{H}$, and can be accomplished efficiently using the histogram-based approach described in [23] and summarized in Table 2. The basic idea is constructing a histogram using the angles estimated from the observations, locating the $L$ highest peaks (setting a guard interval around each peak to avoid double counting), and using the outputs with angles falling inside each of the selected bins to estimate the desired angles of the mixing matrix.

This algorithm has a low computational cost and shows a good performance for two outputs (i.e. $Q=2$ ) and moderate/high SNRs, especially when a large number of samples are available. Unfortunately, although an extension of this method was proposed in [21] for $Q=3$, it is difficult to apply to an arbitrary number of outputs, since it requires constructing a $(Q-1)$ dimensional histogram and searching for the $L$ desired peaks inside it. This implies that storage and computational costs grow exponentially with the number of outputs, thus making this approach unfeasible even for small values of $Q$ (e.g. $Q=4$ or $Q=5$ ).

\section{Random search clustering (RSC) algorithm for arbitrary number of outputs}

\subsection{Parameterization of the mixing matrix: hyperspherical coordinates}

First of all, we note that the parameterization of the mixing matrix introduced in Section 3.2 can be extended to the $Q$ dimensional case by expressing each column of $\mathbf{H}, \mathbf{h}_{\ell}$, using hyperspherical coordinates [21], i.e. using their norm, $\left\|\mathbf{h}_{\ell}\right\|$, and $Q-1$ angles, $\theta_{1, \ell} \in[-\pi, \pi)$ and $\theta_{q, \ell} \in[-\pi / 2, \pi / 2)$ for $2 \leq q \leq Q-1$. Thus, $\mathbf{H}$ can still be expressed as the product of two matrices using (16), where Eq. (17) remains valid for the $L \times L$ diagonal norm matrix, $\boldsymbol{\Lambda}$, with the $L_{2}$ norm of each column defined as

$$
\left\|\mathbf{h}_{\ell}\right\|=\left(\mathbf{h}_{\ell}^{\top} \mathbf{h}_{\ell}\right)^{1 / 2}=\left(\sum_{q=1}^{Q} h_{\ell}^{2}(q)\right)^{1 / 2},
$$

and, instead of using (19) for the $Q \times L$ angular matrix, its $(q, \ell)$-th element $(1 \leq q \leq Q, 1 \leq \ell \leq L)$ is now given by

$\boldsymbol{\Phi}(q, \ell)= \begin{cases}\prod_{m=1}^{Q-1} \cos \left(\theta_{m, \ell}\right), & q=1, \\ \sin \left(\theta_{q-1, \ell}\right) \prod_{m=q}^{Q-1} \cos \left(\theta_{m, \ell}\right), & 2 \leq q \leq Q-1, \\ \sin \left(\theta_{Q-1, \ell}\right), & q=Q,\end{cases}$

with the first angle, $\theta_{1, \ell}$, defined as in the $Q=2$ case, using (20) and (21), and the remaining angles given by

$\theta_{q, \ell}=\arctan \left(\frac{h_{\ell}(q+1)}{\left\|\mathbf{h}_{\ell}(1: q)\right\|}\right)$

for $2 \leq q \leq Q-1$, with $\left\|\mathbf{h}_{\ell}(1: q)\right\|$ denoting the $L_{2}$ norm of the $q$-dimensional vector formed by the first $q$ components of $\mathbf{h}_{\ell}$.

Finally, we notice that this novel parameterization of $\mathbf{h}_{\ell}$ allows us to describe it alternatively using its hyperspherical coordinate vector,

$\psi\left(\mathbf{h}_{\ell}\right)=\left[\left\|\mathbf{h}_{\ell}\right\|, \boldsymbol{\theta}_{\ell}^{\top}\right]^{\top}$,

with $\boldsymbol{\theta}_{\ell}^{\top}=\left[\theta_{1, \ell}, \ldots, \theta_{Q-1, \ell}\right]$ being the angular vector associated to the $\ell$-th column of the mixing matrix. This hyperspherical coordinate vector is used in the sequel for uniquely characterizing $\mathbf{h}_{\ell}$ instead of the Cartesian coordinate vector, $\mathbf{h}_{\ell}=\left[h_{\ell}(1), \ldots, h_{\ell}(Q)\right]^{\top}$.

\subsection{Noiseless case: coordinate vector, colinear set and algorithm}

For the noiseless case, whenever the $\ell$-th source is the only one active, the hyperspherical coordinate vector for $\mathbf{x}_{\ell}[n]$ is given by

$$
\psi\left(\mathbf{x}_{\ell}[n]\right)=\left[\left\|\mathbf{x}_{\ell}[n]\right\|, \varphi\left(\mathbf{x}_{\ell}[n]\right)^{\top}\right]^{\top},
$$

where $\left\|\mathbf{x}_{\ell}[n]\right\|=\left|s_{\ell}[n]\right| \times\left\|\mathbf{h}_{\ell}\right\|$ is the norm of $\mathbf{x}_{\ell}[n]$ and $\boldsymbol{\varphi}\left(\mathbf{x}_{\ell}[n]\right)$ is the angular vector associated to $\mathbf{x}_{\ell}[n]$, with components given by

$\varphi_{1}\left(\mathbf{x}_{\ell}[n]\right)=\arctan \left(\frac{x_{\ell}^{(2)}[n]}{x_{\ell}^{(1)}[n]}\right)$,

for the first angle, and

$\varphi_{q}\left(\mathbf{x}_{\ell}[n]\right)=\operatorname{sign}\left(x_{\ell}^{(1)}[n]\right) \arctan \left(\frac{x_{\ell}^{(q+1)}[n]}{\left\|\mathbf{x}_{\ell}^{(1: q)}[n]\right\|}\right)$.

for the rest of the angles. Comparing (30) and (20) we see that $\varphi_{1}\left(\mathbf{x}_{\ell}[n]\right)=\tilde{\theta}_{1, \ell} \in[-\pi / 2, \pi / 2)$, which is the two quadrant version of $\theta_{1, \ell} \in[-\pi, \pi)$. Regarding the remaining angles, the range adopted for $\varphi_{1}\left(\mathbf{x}_{\ell}[n]\right)$ implies that $h_{\ell}(1) \geq 0$, meaning that $\operatorname{sign}\left(x_{\ell}^{(1)}[n]\right)=\operatorname{sign}\left(s_{\ell}[n]\right), \varphi_{q}\left(\mathbf{x}_{\ell}[n]\right)=\theta_{q, \ell}$, and

$\boldsymbol{\varphi}\left(\mathbf{x}_{\ell}[n]\right)=\tilde{\boldsymbol{\theta}}_{\ell}=\left[\tilde{\theta}_{1, \ell}[n], \boldsymbol{\theta}_{\ell}(2: Q-1)^{\top}\right]^{\top}$.

Eq. (32) implies that the hyperspherical coordinate vector associated to the noiseless observation vectors, $\psi\left(\mathbf{x}_{\ell}[n]\right)$, can be used to identify the hyperspherical coordinate vector of $\mathbf{h}_{\ell}$ up to a global scale factor (i.e. a norm undeterminacy and a quadrant ambiguity in the first angle). Hence, the $\ell$-th cluster that we are searching for is given by

$\mathcal{C}_{\ell}=\left\{n: \mathbf{x}[n]=\mathbf{x}_{\ell}[n]=s_{\ell}[n] \mathbf{h}_{\ell}\right\}=\left\{n: \boldsymbol{\varphi}(\mathbf{x}[n])=\tilde{\boldsymbol{\theta}}_{\ell}\right\}$,

which is the colinear set for the $\ell$-th column of $\mathbf{H}$. Unfortunately, the problem for finding $\mathcal{C}_{\ell}$ using (33) is that $\tilde{\boldsymbol{\theta}}_{\ell}$ is unknown. Nevertheless, in the noiseless case $\tilde{\theta}_{\ell}$ can be easily found by applying a simple random search procedure:

1. Select an observation vector $\mathbf{x}[n] \neq \mathbf{0}$ randomly as candidate basis vector for the $\ell$-th cluster, $\tilde{\mathbf{x}}_{\ell}$.

2. Search the whole data set, adding an observation to the

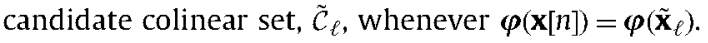


3. If enough colinear observations are found (i.e. if the cardinality of $\tilde{\mathcal{C}}_{\ell}$ is large enough), accept $\tilde{\mathcal{C}}_{\ell}$ as a valid colinear set. Otherwise return to 1 .

Repeating this process $L$ times (taking care not to select candidate vectors belonging to the colinear set of previously accepted basis vectors), the $L$ clusters can be easily identified, as demonstrated in Section 6.2 .

\subsection{Noisy case: approximate coordinate vector, alternative colinear} set and modified algorithm

In the noisy case, whenever only the $\ell$-th source is active, the observations are given by (15). Hence, the hyperspherical coordinate vector is now

$\psi\left(\mathbf{y}_{\ell}[n]\right)=\left[\left\|\mathbf{y}_{\ell}[n]\right\|, \varphi\left(\mathbf{y}_{\ell}[n]\right)^{\top}\right]^{\top}$,

where $\left\|\mathbf{y}_{\ell}[n]\right\|$ is the $L_{2}$ norm of $\mathbf{y}_{\ell}[n]$,

$\left\|\mathbf{y}_{\ell}[n]\right\|=\left(s_{\ell}^{2}[n]\left\|\mathbf{h}_{\ell}\right\|^{2}+2 s_{\ell}[n] \mathbf{h}_{\ell}^{\top} \mathbf{w}[n]+\|\mathbf{w}[n]\|^{2}\right)^{1 / 2}$,

and $\varphi\left(\mathbf{y}_{\ell}[n]\right)$ denotes the angular vector associated to $\mathbf{y}_{\ell}[n]$. Under moderate/high SNR conditions it can be shown (see Appendix A) that the components of $\varphi\left(\mathbf{y}_{\ell}[n]\right)$ are given by the true angles (with $\pi$ radians ambiguity in the first angle, as discussed before) plus their corresponding noise terms:

$\boldsymbol{\varphi}\left(\mathbf{y}_{\ell}[n]\right)=\tilde{\boldsymbol{\theta}}_{\ell}+\mathcal{\Delta} \boldsymbol{\theta}_{\ell}[n]$,

with $\tilde{\boldsymbol{\theta}}_{\ell}$ given by (32) and $\Delta \boldsymbol{\theta}_{\ell}[n]$ the $(\mathrm{Q}-1) \times 1$ angular difference vector, with components given by (A.3) and (A.4)

The key point now is noticing that the variance of all the elements of $\Delta \boldsymbol{\theta}_{\ell}[n]$, i.e. the mean square error (MSE) between the true and the estimated angles, tends to zero as $\sigma_{w}^{2} \rightarrow 0$. Although we cannot prove this theoretically, it can be easily justified empirically. Fig. 4 displays the average MSE (obtained using 1000 simulations) of the four angles associated to the first column of the $5 \times 8$ mixing matrix obtained from (66), i.e.

$\operatorname{MSE}\left(\tilde{\theta}_{q, \ell}\right)=\mathrm{E}\left\{\left(\varphi_{q}\left(\mathbf{y}_{\ell}\right)-\tilde{\theta}_{q, \ell}\right)^{2}\right\}=\mathrm{E}\left\{\left(\Delta \theta_{q, \ell}\right)^{2}\right\}$,

for $1 \leq q \leq Q-1=4$ and $\ell=1$, estimated from the observations as

$\operatorname{MSE}\left(\tilde{\theta}_{q, \ell}\right)=\frac{1}{\left|\mathcal{C}_{\ell}\right|} \sum_{n \in \mathcal{C}_{\ell}}\left(\varphi_{q}\left(\mathbf{y}_{\ell}[n]\right)-\tilde{\theta}_{q, \ell}\right)^{2}$,

in three possible situations: inputs with fixed amplitude equal to one (continuous lines), inputs distributed according to the B-G model with unit variance (dashed lines) and these same inputs using only samples with norm greater than 0.1 for the estimation (dotted lines). The exponential decrease in average MSE as the noise variance decreases can be clearly appreciated for all cases. Moreover, Fig. 4 also shows the need to establish a norm threshold in the observations before estimating the angles, since inputs with low amplitudes are very sensitive to noise and can lead to poor angular estimations.

The previous discussion confirms that the outputs tend to cluster around the directions given by the columns of the mixing matrix for moderate/high SNRs. Hence, we can define the colinear set in a similar way to (33):

$\mathcal{C}_{\ell}=\left\{n: \mathbf{y}[n]=\mathbf{x}_{\ell}[n]+\mathbf{w}[n]\right\}=\left\{n: \varphi(\mathbf{y}[n])=\tilde{\theta}_{\ell}+\Delta \boldsymbol{\theta}_{\ell}[n]\right\}$

The problem, in addition to finding $\tilde{\boldsymbol{\theta}}_{\ell}$ as in the noiseless case, is deciding when $\varphi(\mathbf{y}[n])$ is approximately equal to $\tilde{\boldsymbol{\theta}}_{\ell}$. For this purpose, it is better to use an alternative definition of the colinear set,

$\mathcal{C}_{\ell}=\left\{n: d\left(\varphi(\mathbf{y}[n]), \tilde{\boldsymbol{\theta}}_{\ell}\right) \leq D_{\max }\right\}$,

where $d\left(\varphi(\mathbf{y}[n]), \tilde{\boldsymbol{\theta}}_{\ell}\right)$ denotes a properly selected distance function and $D_{\max }$ is a design parameter of the algorithm. From (40) we notice that, using the $L_{\infty}$ norm of the angular difference as the distance

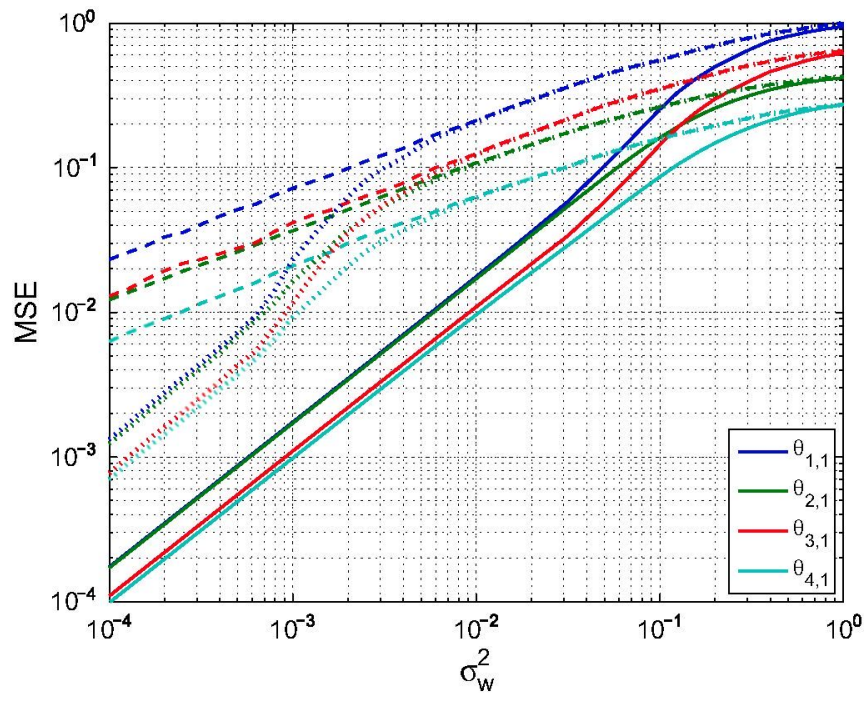

Fig. 4. MSE of the four angles associated to the first column of the $5 \times 8$ mixing matrix given by (66), estimated from the outputs for inputs with fixed amplitudes (continuous lines), distributed according to the B-G model using all the observations (dashed lines) and only observations with norm greater than 0.1 (dotted lines).

function,

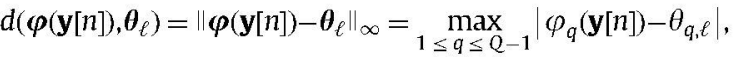

$\mathcal{C}_{\ell}$ is the $(Q-1)$-dimensional generalization of the colinear set $\Pi_{\ell}$ used in the histogram-based algorithm. However, from (A.2) and (A.5) we see that $q+1$ independent input noise samples appear in the estimation of the $q$-th angle. Thus, on average the standard deviation of $\xi_{\ell}^{(q)}[n]$ is $\sqrt{(q+1) / 2}$ times larger than the standard deviation of $\xi^{(1)}[n]$. Hence, we modify the distance function in (41), pre-multiplying the difference vector by a $(Q-1) \times(Q-1)$ diagonal matrix,

$\mathbf{K}_{\varphi}=\operatorname{diag}(1, \sqrt{2 / 3}, \ldots, \sqrt{2 /(q+1)}, \ldots, \sqrt{2 / Q})$,

obtaining the final distance function proposed,

$d\left(\boldsymbol{\varphi}(\mathbf{y}[n]), \boldsymbol{\theta}_{\ell}\right)=\left\|\mathbf{K}_{\varphi}\left(\boldsymbol{\varphi}(\mathbf{y}[n])-\boldsymbol{\theta}_{\ell}\right)\right\|_{\infty}=\max _{1 \leq q \leq Q-1} \frac{\sqrt{2}\left|\varphi_{q}(\mathbf{y}[n])-\theta_{q, \ell}\right|}{\sqrt{q+1}}$,

which is equivalent to using (41) with a maximum distance for the $q$-th element of the difference vector given by $D_{\max } \sqrt{(q+1) / 2}$.

The discussion performed in this section shows that we can follow a similar procedure to the noiseless case for finding the clusters:

1. Select an observation vector $\mathbf{y}[n]$, such that $\|\mathbf{y}[n]\| \geq A_{\min }$, randomly as candidate basis vector for the $\ell$-th cluster, $\tilde{\mathbf{y}}_{\ell}$.

2. Search the whole data set, adding an observation to the potential colinear set, $\tilde{\mathcal{C}}_{\ell}$, whenever $d\left(\varphi(\mathbf{y}[n]), \varphi\left(\tilde{\mathbf{y}}_{\ell}\right)\right) \leq D_{\max }$.

3. If enough colinear observations are found (i.e. if the cardinality of $\tilde{\mathcal{C}}_{\ell}$ is large enough), accept $\tilde{\mathcal{C}}_{\ell}$ as a valid colinear set. Otherwise return to 1 .

As in the noiseless case, repeating this process $L$ times (selecting new candidate vectors with an angular distance at least $\tilde{D}_{\max }$ from previously accepted candidates) we find the desired clusters, as shown in Sections 6.3-6.5. The details of the final clustering approach proposed, called random search clustering (RSC) algorithm, are summarized in Table 3. Once clustering has been performed, the angular estimation can be done as in the histogram-based approach: estimating the $\ell$-th angular vector as the average of all the angular 
Table 3

Random search clustering (RSC) algorithm for identification of the directions of maximum data density.

$$
\begin{aligned}
& \text { For } \ell=1, \ldots, L \text { : } \\
& \text { 1. While a colinear set for } \mathbf{h}_{\ell} \text { is not available, randomly select a candidate } \\
& \text { vector, } \mathbf{y}\left[n_{\ell}\right]=\tilde{\mathbf{y}}_{\ell} \text {, such that } \\
& \left\|\mathbf{y}\left[\boldsymbol{n}_{\ell}\right]\right\|=\left\|\tilde{\mathbf{y}}_{\ell}\right\| \geq A_{\min } \\
& d\left(\boldsymbol{\varphi}\left(\tilde{\mathbf{y}}_{\ell}\right), \boldsymbol{\varphi}\left(\tilde{\mathbf{y}}_{m}\right)\right) \geq \tilde{D}_{\max }
\end{aligned}
$$

for $1 \leq m \leq \ell-1$ and $d\left(\varphi\left(\tilde{\mathbf{y}}_{\ell}\right), \varphi\left(\tilde{\mathbf{y}}_{m}\right)\right)$ given by (43). Initialize the candidate colinear set for $\mathbf{h}_{\ell}$ to this candidate vector, i.e. $\tilde{\mathcal{C}}_{\ell}=\left\{n_{\ell}\right\}$

2. Search through the whole data vector, adding the $n$-th observation to $\tilde{\mathcal{C}}_{\ell}$ if and only if

$$
\begin{aligned}
& \|\mathbf{y}[n]\| \geq A_{\min } \\
& d\left(\varphi(\mathbf{y}[n]), \varphi\left(\tilde{\mathbf{y}}_{\ell}\right)\right) \leq D_{\max }
\end{aligned}
$$

with $d\left(\varphi(\mathbf{y}[n]), \varphi\left(\tilde{\mathbf{y}}_{\ell}\right)\right)$ given by (43). In this case, set $\tilde{\mathcal{C}}_{\ell}=\tilde{\mathcal{C}}_{\ell} \cup\{n\}$.

3. Accept the candidate vector if the cardinality of the candidate colinear set, $\left|\tilde{C}_{\ell}\right|$, is greater or equal than $N_{\min }$. In this case, establish the final colinear set for $\mathbf{h}_{\ell}$ as $\mathcal{C}_{\ell}=\tilde{\mathcal{C}}_{\ell}$ and obtain the estimate of the $\ell$-th angular vector, $\hat{\boldsymbol{\theta}}_{\ell}$, using (44). Otherwise, reject it and return to step 1

vectors belonging to the $\ell$-th colinear set,

$$
\hat{\boldsymbol{\theta}}_{\ell}=\frac{1}{\left|\mathcal{C}_{\ell}\right|} \sum_{n \in \mathcal{C}_{\ell}} \boldsymbol{\varphi}(\mathbf{y}[n]) .
$$

\subsection{Computational cost}

The computational cost of the RSC algorithm grows linearly with the number of samples and columns of the mixing matrix, i.e. its computational cost is $\mathcal{O}(L N)$. The practical implementation of the algorithm requires a pre-processing step where all the output samples are checked. Those with $\|\mathbf{y}[n]\|<A_{\min }$ are discarded, whereas the angles associated to the remaining samples are calculated and stored. The algorithm works with these angles, using them to find new candidate vectors and accepting or discarding them through simple comparisons, as described in Table 3. Hence, the constant multiplying the $L N$ term in the computational cost ultimately depends on the number of useful samples, $N_{u}=\rho N=(1-\eta) \eta^{L-1} N<N$ as defined in Section 5.3, as well as the number of trials required for obtaining all the valid directions, $\kappa L \geq L$. This means that the computational cost is proportional to $\rho \kappa L N<L N$ for large sparsity factors and moderate/high SNRs. For example, for $Q=5, L=8, \eta=0.9, N=10000$ and $\mathrm{SNR}=60 \mathrm{~dB}$, we have $\rho \approx 0.0478$ and an average value of $\kappa \approx 6.73$ (obtained for 1000 simulations with randomly generated mixing matrices). Hence, $\rho \kappa \approx 0.3217$ and the computational cost of the RSC algorithm is roughly proportional to $L N / 3$.

\section{Parameter selection for the RSC algorithm}

\subsection{Norm threshold $\left(A_{\min }\right)$}

The purpose of the norm threshold is discriminating between noise and signal vectors. It is used at two stages of the algorithm: initially in the random candidate vector selection stage, and later on for accepting or discarding vectors potentially colinear with the candidate. The threshold selection problem can be formulated as a binary hypothesis testing problem. On the one hand, the null or noise hypothesis, $\mathcal{H}_{0}$, considers the $n$-th observation only due to noise, i.e. $\mathbf{y}[n]=\mathbf{w}[n]$, and, assuming $\sigma_{w_{q}}^{2}=\sigma_{w}^{2}$ for $1 \leq q \leq Q, p\left(\mathbf{y}[n] \mid \mathcal{H}_{0}\right)=$
$\mathcal{N}\left(\mathbf{0}, \sigma_{w}^{2} \mathbf{I}\right)$. On the other hand, the signal hypothesis, $\mathcal{H}_{1}$, supposes that one or more inputs are active, i.e. $\mathbf{y}[n]=\mathbf{x}[n]+\mathbf{w}[n]$.

Unfortunately, an expression for $p\left(\mathbf{y}[n] \mid \mathcal{H}_{1}\right)$ cannot be obtained without assuming a specific PDF for the inputs. Hence, in the sequel we concentrate on the null hypothesis and solve the problem using the Neyman-Pearson criterion [22]. Moreover, instead of working with the $Q$-dimensional vector $\mathbf{y}[n]$, we use the one-dimensional squared norm of the observations, $\lambda=\mid \mathbf{y}[n] \|^{2}$, which is a sufficient statistic for this problem. The PDF associated to the squared sum of $Q$ independent normal random variables with zero mean and unit variance is a chi square with $Q$ degrees of freedom, $\chi_{Q}^{2}(x)$ [43]. Hence, the PDF for the null hypothesis is given by

$$
\begin{aligned}
p_{A}\left(\lambda \mid \mathcal{H}_{0}\right) & =\frac{1}{\sigma_{w}^{2}} \chi_{Q}^{2}\left(\frac{\lambda}{\sigma_{w}^{2}}\right) \\
& =\frac{1}{2^{Q / 2} \sigma_{w}^{2} \Gamma(Q / 2)}\left(\frac{\lambda}{\sigma_{w}^{2}}\right)^{Q / 2-1} \exp \left(-\frac{\lambda}{2 \sigma_{w}^{2}}\right) u\left(\frac{\lambda}{\sigma_{w}^{2}}\right),
\end{aligned}
$$

where $\Gamma(\mathrm{Q} / 2)$ is the gamma function [44], and the false alarm probability is

$P_{f a}=1-\int_{0}^{A_{\min }^{2}} p_{A}\left(\lambda \mid \mathcal{H}_{0}\right) \mathrm{d} \lambda=1-P\left(A_{\min }^{2} /\left(2 \sigma_{w}^{2}\right) ; Q / 2\right)$,

with $P(x ; a)$ denoting the incomplete gamma function [44].

Finding an analytical expression for the integral in (46) and any value of $Q$ is not possible, so we cannot obtain a closed-form expression for $A_{\min }$ as a function of $P_{f a}$ for the general case. ${ }^{2}$ However, since $P(x ; a)$ is a strictly increasing function of $x$, the cost function,

$J\left(E_{\min }\right)=P\left(E_{\min } /\left(2 \sigma_{w}^{2}\right) ; Q / 2\right)-\left(1-P_{f a}\right)$

with $E_{\min }=A_{\min }^{2}$, has exactly one zero, located at the desired value of $E_{\min }$, which can be easily found performing a few iterations of Newton's method [46], since $P(x ; a)$ is differentiable [44], and a closed-form expression can be provided for the derivative of (47):

$\frac{\mathrm{d} J\left(E_{\min }\right)}{\mathrm{d} E_{\min }}=\frac{1}{2 \sigma_{w}^{2} \Gamma(Q / 2)}\left(\frac{E_{\min }}{2 \sigma_{w}^{2}}\right)^{Q / 2-1} \exp \left(-\frac{E_{\min }}{2 \sigma_{w}^{2}}\right)$.

Fig. 5 shows the norm threshold obtained using Newton's method (initialized with $E_{\min }(0)=10 \sigma_{w}^{2}$ ) for $Q=2, Q=3$ and $Q=5$, altogether with the true value for $Q=2$. In all cases $A_{\min }$ decays exponentially with the SNR and, for $Q=2$, the value obtained using Newton's method is very close to the exact value, with a relative error around 0.03 regardless of the SNR.

\subsection{Colinearity thresholds $\left(D_{\max }\right.$ and $\left.\tilde{D}_{\max }\right)$}

The colinearity thresholds are used for two purposes in the RSC algorithm: selecting a new candidate vector which is not colinear with previously accepted candidates using $\tilde{D}_{\max }$, and deciding whether a new data vector belongs to the colinear set of a candidate or not using $D_{\max }$. In this sense, their role is similar to that of the bin size, $\Delta \theta$, and the number of guard samples, $N_{z}$, in the histogram-based algorithm described in Section 3.2. Therefore, we set $D_{\max }=\Delta \theta / 2=\pi /\left(2 N_{b}\right)$, where $N_{b}$ would be the number of bins per output dimension of the equivalent $(Q-1)$ dimensional histogram, and $\tilde{D}_{\max }=\Delta \tilde{\theta} / 2=\left(2 N_{z}+1\right) \pi /\left(2 N_{b}\right)$, with $N_{z}$ being the number of bins set to zero per output dimension of the equivalent $(Q-1)$-dimensional histogram to avoid double

\footnotetext{
${ }^{2}$ Analytical expressions for the integral in (46) can only be found when $Q / 2$ is an integer number [45], but finding a closed-form expression for $A_{\min }$ as a function of $P_{f a}$ is not possible even in this case, except for $Q=2$, where we have $A_{\min }=\sqrt{-2 \sigma_{w}^{2} \ln \left(P_{f a}\right)}$.
} 


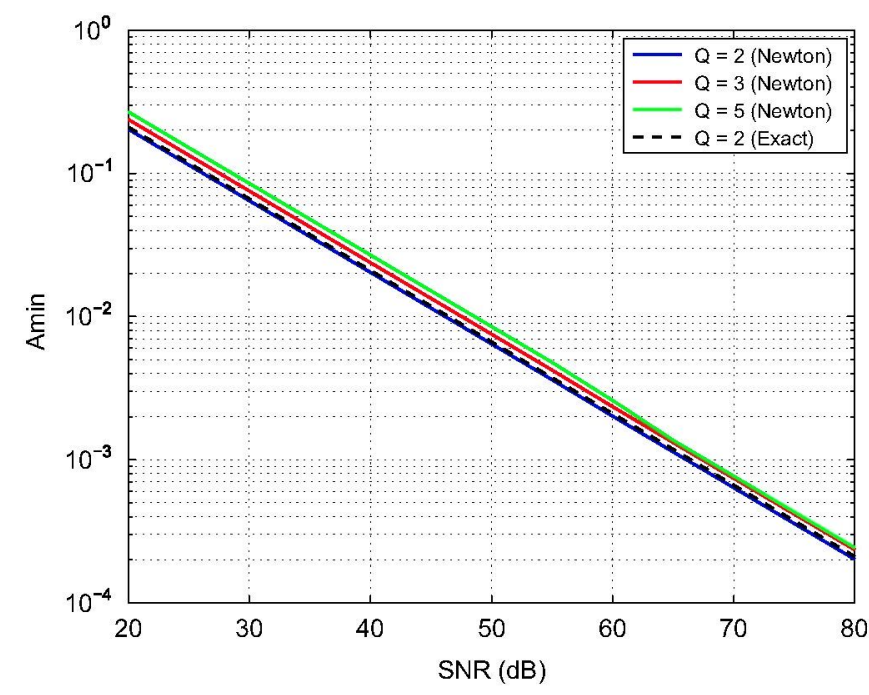

Fig. 5. Norm threshold, $A_{\min }$, as a function of the number of outputs, $Q$ and the SNR. Values obtained using Newton's method, and the true value for $Q=2$.

counting of a single valid direction. ${ }^{3}$ These choices ease the comparison with the histogram-based estimator for $Q=2$ and provide a straightforward geometric characterization of the colinear and exclusion sets.

\subsection{Minimum cardinality of the colinear set $\left(N_{\min }\right)$}

The minimum cardinality of the colinear set, $N_{\min }$, is used to determine whether a candidate basis vector is accepted or not. In this section we discuss two alternative approaches for obtaining a PDF-independent value for this parameter. In Section 5.3.1 we obtain a first value for $N_{\min }$, denoted $N_{h i g h}$, taking into account only the distribution of the number of samples colinear with each of the columns of $\mathbf{H}$. Then, in Section 5.3.2 we obtain an alternative value for $N_{\min }$, denoted $N_{\text {low }}$, considering only the distribution of null samples. Unfortunately, the first threshold is too high for moderate/low SNR situations, whereas the second one is always too low. Hence, in Section 5.3.3 we show how a robust threshold can be obtained as a convex combination of those two thresholds using a factor that depends on the SNR.

\subsubsection{Upper bound for the threshold $\left(N_{\text {high }}\right)$}

In the sequel we assume that the sparsity factor for all the sources is the same, $\eta$, and define a colinearity factor, $\rho$, as the probability that a given observation belongs to the colinear set of the $\ell$-th output $(1 \leq \ell \leq L)$ :

$\rho=\operatorname{Pr}\left\{\mathbf{x}[n] \in \mathcal{C}_{\ell}\right\}=\operatorname{Pr}\left\{\llbracket(\mathbf{s}[n])=\left[\mathbf{0}_{\ell-1}^{\top}, 1, \mathbf{0}_{L-\ell}^{\top}\right]^{\top}\right\}=(1-\eta) \eta^{L-1}$,

where $\square(s[n])=\left[\square\left(s_{1}[n]\right), \ldots, \square\left(s_{\ell}[n]\right), \ldots, \square\left(s_{I}[n]\right)\right]^{\top}$ is the vector obtained applying the indicator function to each component of $\mathbf{s}[n]$ independently. This factor could serve as the basis for a heuristic threshold, since the average number of useful samples for the $\ell$-th source (i.e. the average number of samples where the $\ell$-th source is the only one active) is given by $N_{u}=\rho N=N(1-\eta) \eta^{L-1}$. Hence, we could simply set $N_{\min }=C N_{u}$ for some $0<C \leq 1$. Fig. 6 displays $\rho$ as a function of $\eta$ and $L$, showing that the maximum number of useful samples is obtained for a sparsity factor that depends on the number of inputs, and how this value decreases as $L$ increases.

\footnotetext{
${ }^{3}$ Note that this $(Q-1)$-dimensional histogram would require a total number of $N_{b}^{Q-1}$ bins, thus making it unfeasible for practical use even for small values of $Q$.
}

In order to ensure a desired level for the probability of not detecting a valid candidate direction, we must analyze the distribution associated to the number of samples colinear with a column of the mixing matrix. For this purpose, we define

$\Xi_{\ell}[n]= \begin{cases}1, & \square(\mathbf{s}[n])=\left[\mathbf{0}_{\ell-1}^{-}, 1, \mathbf{0}_{L-\ell}^{\top}\right]^{\top}, \\ 0 & \text { otherwise. }\end{cases}$

For a fixed value of $n, \Xi_{\ell}[n]$ is a Bernoulli variable with probability of success equal to $\rho$, i.e. $\Xi_{\ell}[n] \sim \mathcal{B}_{1}(\rho)$, whereas, as a function of $n$ and assuming independence across trials (i.e. samples of the outputs), ${ }^{4} \Xi_{\ell}[n]$ is a Bernoulli process [43]. Therefore, the number of successes in $N$ trials (i.e. the number of useful samples for the $\ell$-th source) is given by

$\mathcal{S}_{\ell}^{(1)}[N]=\sum_{n=1}^{N} \Xi_{\ell}[n]$,

which follows a binomial distribution, i.e. $\mathcal{S}_{\ell}^{(1)}[N] \sim \mathcal{B}_{N}(\rho)$, with an expected value $\mu_{\mathcal{H}_{1}}=N_{u}=\rho N$ and a variance $\sigma_{\mathcal{H}_{1}}^{2}=(1-\rho)$ $N_{u}=\rho(1-\rho) N$.

Using $\mathcal{S}_{\ell}^{(1)}[N]$ the problem has been reduced once more to a binary hypothesis testing problem that can be solved again using the Neyman-Pearson criterion for a desired non-detection probability, $P_{n d}$ :

$$
\begin{aligned}
P_{n d} & =\operatorname{Pr}\left\{\mathcal{S}_{\ell}^{(1)}[N]<N_{\text {high }}\right\}=\sum_{k=0}^{N_{\text {high }}-1}\left(\begin{array}{l}
N \\
k
\end{array}\right)(1-\rho)^{k} \rho^{N-k} \\
& =1-I_{\rho}\left(N_{\text {high }}, N-N_{\text {high }}+1\right),
\end{aligned}
$$

where $I_{x}(a, b)$ denotes the incomplete beta function [44]. Unfortunately, we cannot obtain a closed-form solution for $N_{\text {high }}$ as a function of $P_{n d}$, but we can formulate it as a simple one-dimensional integer optimization problem,

$N_{\text {high }}=\underset{1 \leq N^{*} \leq N}{\operatorname{argmax}} I_{\rho}\left(N^{*}, N-N^{*}+1\right)$,

subject to

$I_{\rho}\left(N_{\text {high }}, N^{*}-N_{\text {high }}+1\right) \geq 1-P_{\text {nd }}$,

which can be easily solved taking increasing values of $N^{*}$ (starting at $N^{*}=1$ ) and selecting $N_{\text {high }}$ as the largest value of $N^{*}$ such that (54) is still fulfilled.

\subsubsection{Lower bound for the threshold ( $N_{\text {low }}$ )}

Alternatively, we may consider the noise samples falling within the colinear distance of a given direction, establish a maximum false alarm rate and obtain a lower bound for the threshold using the Neyman-Pearson criterion. The probability of having an observation which is only due to noise and belongs to the colinear set of the $\ell$-th column of $\mathbf{H}$ is given by

$$
\begin{aligned}
\varrho & =\operatorname{Pr}\left\{\mathbf{y}[n]=\mathbf{w}[n] \in \mathcal{C}_{\ell}\right\} \\
& =\operatorname{Pr}\{\square(\mathbf{s}[n])=\mathbf{0}\} \times \operatorname{Pr}\left\{\mathbf{w}[n] \in \mathcal{C}_{\ell}\right\}=\frac{\eta^{L}}{N_{b}^{Q-1}} \prod_{q=1}^{Q-1} \sqrt{\frac{q+1}{2}},
\end{aligned}
$$

where we have taken into account the distance function proposed in Section 4.3, the parameter $D_{\max }=\pi /\left(2 N_{b}\right)$ defined in Section 5.2 , and the uniform angular distribution of noise samples. Now we follow an identical procedure to the previous section, defining $\Psi_{\ell}[n]= \begin{cases}1, & \mathbf{y}[n]=\mathbf{w}[n] \in \mathcal{C}_{\ell}, \\ 0 & \text { otherwise, }\end{cases}$

${ }^{4}$ This independence condition is strictly fulfilled by an instantaneous mixture but not by a convolutive mixture. However, the memory of $\Xi_{\ell}[n]$ (related to $\max L_{r}$ ) is typically very small compared to the data size, $N$, and thus it can be considered a good approximation. 


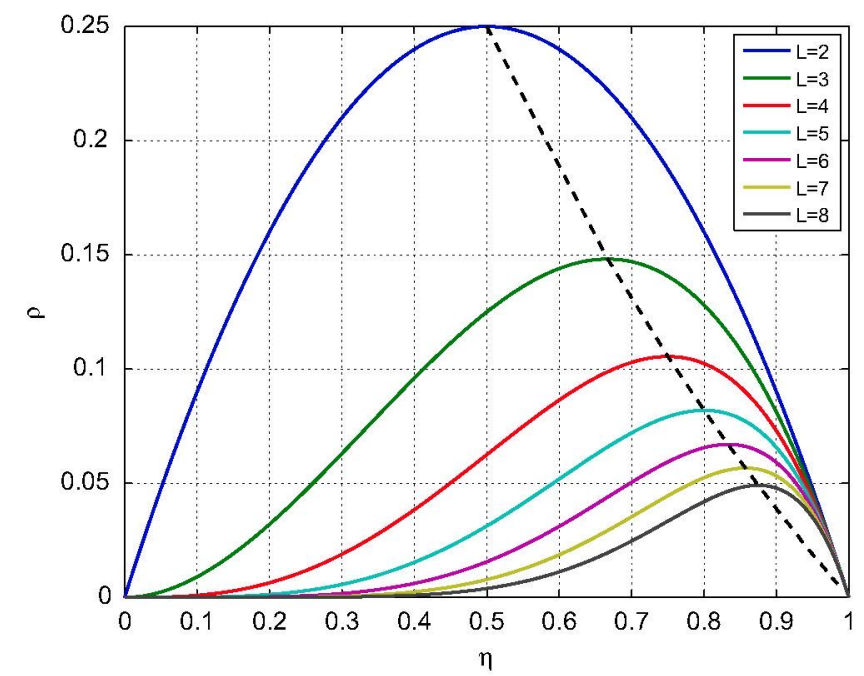

Fig. 6. Colinearity factor, $\rho$, as a function of the sparsity factor, $\eta$, and the number of inputs, $L$. The dashed black line shows how the position of the maximum value of $\rho$ increases as a function of $L$.

which follows a Bernoulli distribution with success probability $\varrho$ given by (55). Assuming independence across trials of $\Psi_{\ell}[n]$ we obtain again a Bernoulli process, with the sum

$\mathcal{S}_{\ell}^{(0)}[N]=\sum_{n=1}^{N} \Psi_{\ell}[n]$

following a binomial distribution, i.e. $\mathcal{S}_{\ell}^{(0)}[N] \sim \mathcal{B}_{N}(\varrho)$, with an expected value $\mu_{\mathcal{H}_{0}}=N_{w}=\varrho N$ and a variance $\sigma_{\mathcal{H}_{0}}^{2}=(1-\varrho) N_{w}=$ $\varrho(1-\varrho) N$. Hence, we have another binary hypothesis testing problem that can be solved using the Neyman-Pearson criterion for a desired false alarm probability, $P_{f a}$ :

$P_{f a}=\operatorname{Pr}\left\{\mathcal{S}_{\ell}^{(0)}[N] \geq N_{\text {low }}\right\}=I_{\varrho}\left(N_{\text {low }}, N-N_{\text {low }}+1\right)$.

Once more we cannot obtain an analytical expression for $N_{\text {low }}$ as a function of $P_{f a}$, but formulating (52) as a one-dimensional integer optimization problem,

$N_{\text {low }}=\underset{1 \leq N^{*} \leq N}{\operatorname{argmax}} I_{\varrho}\left(N^{*}, N-N^{*}+1\right)$,

subject to

$I_{\varrho}\left(N_{\text {low }}, N^{*}-N_{\text {low }}+1\right) \leq P_{f a}$,

we can easily solve it in the same way as before: taking increasing values of $N^{*}$ (starting at $N^{*}=1$ ) and selecting $N_{\text {low }}$ as the largest value of $N^{*}$ such that (60) is still fulfilled.

\subsubsection{Final threshold: convex combination of $N_{\text {low }}$ and $N_{\text {high }}$}

The colinearity thresholds obtained in the previous two sections have only considered the distribution of the number of samples colinear with a given column of $\mathbf{H}$, for a given number of inputs and sparsity factor, both in the signal and the noise case. However, there are several factors that we cannot take into account without assuming a specific distribution for the inputs. On the one hand, by assuming that all the samples with $\Xi_{\ell}[n]=1$ belong to $\mathcal{C}_{\ell}$, we are ignoring those samples with $\left\|\mathbf{y}_{\ell}[n]\right\|<A_{\min }$ and/or $d\left(\mathbf{y}_{\ell}[n], \tilde{\mathbf{y}}_{\ell}\right)>D_{\max }$ in the calculus of $N_{h i g h}$, which can then be considered an upper bound for the true threshold. On the other hand, by considering only false alarms due to noise, we are neglecting false alarms caused by combinations of two or more columns of $\mathbf{H}$ in the derivation of $N_{\text {low }}$, which becomes a lower bound for the true threshold. Moreover, the thresholds obtained do not depend on the noise level, whereas it can be easily verified empirically that the SNR does have a great influence in the cardinality of the estimated colinear set. Hence, in this section we propose to find the colinearity threshold, $N_{\min }$, as a convex combination of those two thresholds,

$N_{\min }=(1-\alpha) N_{\text {low }}+\alpha N_{\text {high }}$,

with the factor $\alpha(0 \leq \alpha \leq 1)$ depending on the SNR:

$\alpha=\left[1+10^{-\left(\mathrm{SNR}(\mathrm{dB})-\mathrm{SNR} R_{0}(\mathrm{~dB})\right) / 10}\right]^{-1}$,

where $S N R_{0}(\mathrm{~dB})$ is a parameter that has to be adjusted empirically as a function of the number of inputs. For low SNRs, i.e. $S N R(\mathrm{~dB}) \ll S N R_{0}(\mathrm{~dB}), \alpha \rightarrow 0$ and $N_{\min } \rightarrow N_{\text {low }}$, whereas for high SNRs, i.e. $S N R(\mathrm{~dB}) \gg S N R_{0}(\mathrm{~dB}), \alpha \rightarrow 1$ and $N_{\min } \rightarrow N_{\text {high }}$, as can be seen in Fig. 7. This is the typical behaviour of a sigmoidal curve, frequently used in neural networks, and follows from the use of the well-known logistic function, $f(x)=(1+\exp (-\beta x))^{-1}$ with $x=S N R(\mathrm{~dB})-S N R_{0}(\mathrm{~dB})$ and $\beta=\ln (10) \approx 2.3$, for the factor $\alpha$.

\subsection{Parameter interrelation}

In the previous sections we have described a way of selecting the parameters for the RSC algorithm $\left(A_{\min }, D_{\max }, \tilde{D}_{\max }\right.$ and $\left.N_{\min }\right)$ separately, assuming a minimal amount of information: additive white Gaussian noise with variance $\sigma_{w}^{2}$, individual samples for the sources distributed according to (8) (without supposing any particular active PDF), and knowledge of the sparsity factor, $\eta$. Unfortunately, all the parameters are interrelated, preventing this parameter selection approach from being globally optimum. Here we briefly discuss how the different parameters influence each other

First of all, we note that the Neyman-Pearson approach described in Section 5.1 for selecting the norm threshold guarantees the desired false alarm rate for noise samples (i.e. the probability of accepting an invalid noise sample), $P_{f a}$, but ignores the false alarms caused by two or more sources being active simultaneously. Moreover, this approach does not care about the non-detection probability (i.e. the probability of discarding a valid sample), $P_{n d}$, and many valid samples may be discarded, as shown by (67)-(69). Therefore, the number of useful samples for the algorithm will be typically lower than expected, and thus $N_{\text {min }}$ should be decreased by a factor dependent on $A_{\min }$ to compensate this fact. Unfortunately, unless a specific active PDF is assumed, this can only be corrected heuristically. Besides, we have found that, for the high sparsity factors typically considered and moderate values of $P_{f a}$ and $P_{n d}$, the decrease in the number of useful samples is not too severe and the algorithm shows a good performance without any modification.

The second set of parameters, $D_{\max }$ and $\tilde{D}_{\max }$, correspond to the colinearity thresholds, which may be selected independently from the remaining parameters, as described in Section 5.2. The parameter $\tilde{D}_{\max }$ is only used to avoid double counting of a valid direction, so we simply fix it to a reasonable value (i.e. a minimum angular distance). The choice of $D_{\max }$ is more delicate, since it influences the number of useful samples available and thus the choice of $N_{\min }$. Selecting $D_{\max }$ too small may result in many valid samples being discarded in noisy situations, whereas selecting it too large may prevent the identification of two close directions and increases the probability of accepting invalid noise samples. The optimum value for this parameter depends on the active distribution of the sources as well as the noise level, being hard to find even with perfect knowledge of the sources' PDF. Hence, we set it to a reasonable value and adjust $N_{\min }$ as a function of the SNR instead.

Finally, the minimum cardinality of the colinear set, $N_{\min }$, is probably the most important parameter, since it is directly influenced by the selection of $A_{\min }$ and $D_{\max }$, and its choice is 


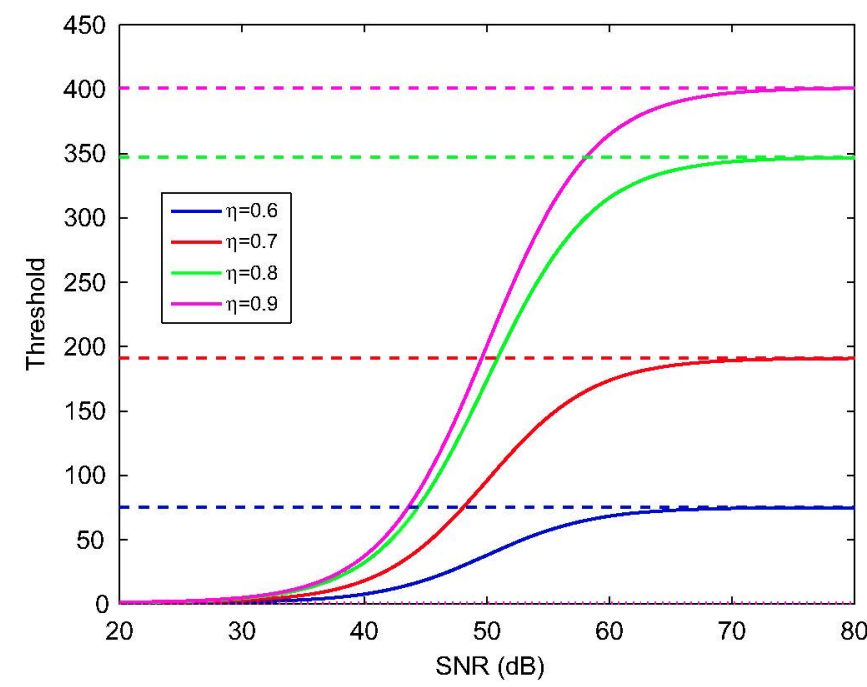

Fig. 7. Colinearity thresholds ( $N_{\text {low }}$ dotted line, $N_{\text {high }}$ dashed line, and $N_{\text {min }}$ continuous line) as a function of the SNR and the sparsity factor for $Q=5, L=8$, $S N R_{0}(\mathrm{~dB})=50$ and $N=10000$.

critical for the good performance of the algorithm: setting it to a very low value results in random invalid directions being accepted, whereas using a very high level means that some valid directions may never be found. Throughout the simulations we have discovered that the method described in Section 5.3 provides a good performance, but the choice of the parameter $S N R_{0}$ is critical. This issue shall be further studied in the future.

\section{Simulations}

\subsection{Mixing matrices}

This section describes the mixing matrices used in the simulations. Since the RSC algorithm focuses on the estimation of the directions of the clusters (i.e. the angular matrix $\boldsymbol{\Phi}$ ), the amplitudes for all the mixing matrices used in the simulations are set to one for all the sources (i.e. $\boldsymbol{\Lambda}=\mathbf{I}$, with $\mathbf{I}$ denoting the $L \times L$ identity matrix), implying that $\mathbf{H}=\boldsymbol{\Phi}$. In the sequel we consider three cases: $Q=2$, where the RSC method is compared with the histogram-based approach; $Q=3$ and $Q=5$, where the histogram-based approach, as described in [23], cannot be applied. In all cases the results shown in the simulations are obtained averaging over 20 random matrices, generated choosing the angles uniformly inside $[0, \pi]$ with a restriction on the minimum angular distance between any two columns of the mixing matrix similar to the one imposed in [20]. This restriction is necessary to avoid ill-conditioned mixing matrices, which cannot be properly identified by any BSS algorithm, and amounts to discarding those matrices such that

$\max _{1 \leq q \leq Q-1}\left|\theta_{q, \ell}-\theta_{q, r}\right|<\Delta \theta_{\min }$

for $1 \leq \ell, r \leq L, \ell \neq r$, and $\Delta \theta_{\min }=\pi / 30$ when $Q=2$ (i.e. $1 / 3$ the minimum angular distance required in [20]) and $\Delta \theta_{\min }=\pi / 10$ for $Q=3$ and $Q=5$ (i.e. the same minimum angular distance required in [20]).

\subsection{Synthetic data: noiseless instantaneous case}

\subsubsection{Two outputs}

In this section we illustrate the performance of the RSC algorithm for $Q=2$ and $L=3$ in the absence of noise (i.e. $\sigma_{w}^{2}=0$, or equivalently, $S N R(\mathrm{~dB})=\infty$ ), comparing it with the histogrambased approach, for inputs distributed according to the B-G model with $\sigma_{f}^{2}=1$ and a mixing matrix constructed using an angular vector $\theta=[9 \pi / 40, \pi / 4,101 \pi / 240]^{\top}$ :

$\mathbf{H}_{1}=\boldsymbol{\Phi}_{1}=\left[\begin{array}{c}\cos (\boldsymbol{\theta}) \\ \sin (\boldsymbol{\theta})\end{array}\right] \approx\left[\begin{array}{lll}0.7604 & 0.7071 & 0.2462 \\ 0.6494 & 0.7071 & 0.9692\end{array}\right]$

This matrix is designed to obtain the two extreme possible situations for the histogram-based clustering algorithm with $N_{b}=180$ (an angle falling exactly in the middle of a bin, $\theta_{1,1}=9 \pi / 40$, and an angle falling just in the frontier between two bins, $\theta_{1,2}=\pi / 4$ ), as well as an angle mid-way between these two situations, $\theta_{13}=101 \pi / 204$.

For the RSC algorithm, following the parameter selection approach described in Section 5 we obtain $A_{\min }=0$ for any $P_{f a}$, and $N_{\min }=N_{\text {high }}$, since $\alpha \rightarrow 1$ as $S N R(\mathrm{~dB}) \rightarrow \infty$, with $N_{\text {high }}$ corresponding to $P_{n d}=10^{-4}$. Moreover, we take $\tilde{D}_{\max }=\pi / 180$ for the minimum distance between two valid directions and, since the angular distance between two observations belonging to $\mathcal{C}_{\ell}$ is always zero in the noiseless case, theoretically we could set $D_{\max }=0$. However, we take $D_{\max }=10^{-9}$ to account for numerical errors in the floating point representation of the simulation software. Using these parameters, the RSC algorithm always obtains a perfect estimation for all the unknown angles defining the columns of $\mathbf{H}_{1}$ (with an MSE around $300 \mathrm{~dB}$, only limited by the resolution of the simulation software) and all the variables tested: $0.5 \leq \eta \leq 0.95$ (with a step size equal to 0.05 ) and $N \in\{1000,2000,5000,10000\}$.

Regarding the histogram-based algorithm, we set $N_{b}=180$ and $N_{z}=1$, obtaining a bin size $\Delta \theta=\pi / 180$ and an exclusion set with a width of $\pm 3 \pi / 360$ rad around the centre of each selected bin. The performance of the histogram using these settings is shown in Fig. 8 for $N=5000$ as a function of $\eta$, both separately for each angle and globally for the whole angular vector. Unlike the RSC algorithm, which provides a uniform performance for all the angles and variables tested, the results for the histogram-based algorithm show a large dependence on the relative position of the true angle inside a bin, as well as on the sparsity factor, $\eta$. Similar results have been obtained for other mixing matrices, leading us to conclude that, in the noiseless case, the RSC algorithm is much more robust than the histogram-based approach, since its performance does not depend on the true value of the angle, the sparsity factor or the number of data points.

\subsubsection{More than two outputs}

For $Q>2$ the histogram-based approach becomes unfeasible. Hence, in this section we just analyse the performance of the noiseless RSC algorithm for $Q=3$ and $L=5$, as well as $Q=5$ and $L=8$. In both cases we use again the B-G model with $\sigma_{f}^{2}=1$ for the inputs and the mixing matrices are obtained from a single realization of the random angle generation mechanism described in Section 6.1, using Matlab's rat function afterwards to obtain a simple rational approximation for the final angles. In particular, the angular vectors obtained for the $(Q, L)=(3,5)$ case are

$$
\theta_{1}=[524 \pi / 923,249 \pi / 302,197 \pi / 367,122 \pi / 241,48 \pi / 83]^{\top},
$$

$\boldsymbol{\theta}_{2}=[395 \pi / 588,111 \pi / 617,110 \pi / 961,287 \pi / 776,253 \pi / 464]^{\top}$,

and the corresponding mixing matrix, $\mathbf{H}_{2}=\mathbf{\Phi}_{2}$, is constructed using (26) with the angular vectors given by $(65)$. For the $(Q L)=(5,8)$ case we can group the four angular vectors in a matrix, 


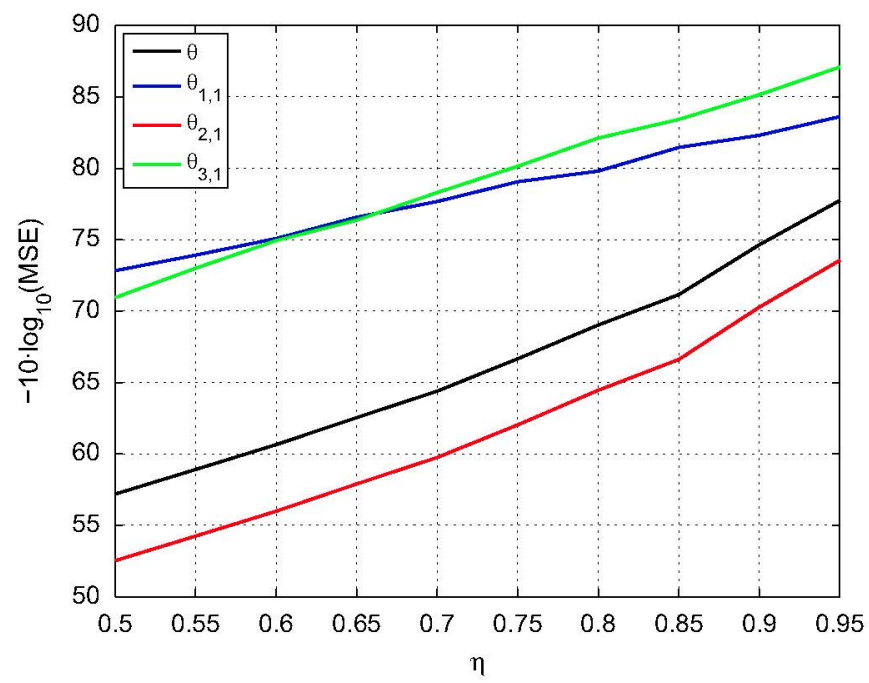

Fig. 8. MSE obtained in the noiseless case estimating the matrix in (64) with the histogram using $N=5000, N_{b}=180$ and $N_{z}=1$.

Table 4

Failure situations of the RSC algorithm for the $(Q L)=(5,8)$ case.

\begin{tabular}{lcccc}
\hline$N$ & 1000 & 1000 & 1000 & 2000 \\
$\eta$ & 0.5 & 0.55 & 0.6 & 0.5 \\
$N_{\text {min }}$ & 0 & 0 & 1 & 0 \\
\hline
\end{tabular}

$\boldsymbol{\Theta}=\left[\begin{array}{l}\boldsymbol{\theta}_{1} \\ \boldsymbol{\theta}_{2} \\ \boldsymbol{\theta}_{3} \\ \boldsymbol{\theta}_{4}\end{array}\right]=\left[\begin{array}{cccccccc}-\frac{53 \pi}{291} & \frac{143 \pi}{304} & \frac{40 \pi}{133} & -\frac{113 \pi}{436} & -\frac{100 \pi}{281} & -\frac{65 \pi}{246} & -\frac{7 \pi}{66} & \frac{269 \pi}{552} \\ -\frac{3 \pi}{400} & -\frac{23 \pi}{362} & -\frac{37 \pi}{132} & \frac{3 \pi}{43} & -\frac{68 \pi}{1495} & \frac{61 \pi}{378} & \frac{281 \pi}{614} & \frac{191 \pi}{516} \\ -\frac{95 \pi}{454} & \frac{136 \pi}{803} & -\frac{23 \pi}{149} & -\frac{156 \pi}{463} & \frac{-194 \pi}{415} & \frac{209 \pi}{923} & -\frac{92 \pi}{429} & \frac{91 \pi}{424} \\ \frac{74 \pi}{809} & -\frac{85 \pi}{172} & -\frac{25 \pi}{76} & \frac{100 \pi}{569} & \frac{50 \pi}{211} & -\frac{153 \pi}{512} & -\frac{188 \pi}{725} & \frac{12 \pi}{61}\end{array}\right]$,

constructing the associated mixing matrix, $\mathbf{H}_{3}=\boldsymbol{\Phi}_{3}$, as before: using (26) with the angles given by (66).

In this case, using the same settings as in the previous section, we notice that the RSC algorithm either obtains a perfect estimation (with an MSE around $300 \mathrm{~dB}$ again) or does not work at all (i.e. the MSE obtained is close to $O \mathrm{~dB}$ ). The reason for this behaviour is that, for some combinations of $\eta$ and $N$, the number of useful samples, $N_{u}$, may be too low. Thus, the colinearity threshold, $N_{\min }=N_{\text {high }}$, which is a nonlinear function of $N_{u}$, is too low and random directions are very likely to be accepted as valid. Table 4 summarizes the situations where the RSC algorithm fails, altogether with the associated value of $N_{\min }$. In all the cases $N_{\text {min }}$ goes down to 0 or 1 , meaning that any non-zero observation not colinear with previously detected directions is accepted as a new valid basis vector. As soon as this colinearity threshold is greater than one, the algorithm works perfectly in the noiseless situation, as observed in the $(Q L)=(5,8)$ case where for $N=1000$ and $\eta=0.65$ we already get $N_{\min }=N_{\text {high }}=4$, whereas for $N=2000$ and $\eta=0.55$ we obtain $N_{\min }=N_{\text {high }}=2$. In both cases the RSC algorithm shows an excellent performance, attaining again an MSE around $300 \mathrm{~dB}$.

\subsection{Synthetic data: noisy instantaneous case}

\subsubsection{Two outputs}

In this section we compare the performance of the RSC algorithm with that of the histogram when the observations are corrupted by additive white Gaussian noise. For the RSC algorithm, we first set $P_{f a}=10^{-2}$ (both for $A_{\min }$ and $\left.N_{\min }\right), P_{n d}=10^{-4}, N_{b}=180, N_{z}=1$ and
$S N R_{0}(\mathrm{~dB})=40$. Then, following again the parameter selection procedure of Section 5 , we obtain $D_{\max }=\pi / 360$ and $\tilde{D}_{\max }=\pi / 120$ in all cases, as well as $A_{\min }$ and $N_{\min }$ for each noise level and sparsity factor. Regarding the histogram-based algorithm, we also use $N_{b}=180$ and $N_{z}=1$ to allow for a fair comparison.

First of all, we show an example of the typical behaviour of both algorithms using the mixing matrix given by (64) and the B-G model for the sources. Fig. 9 compares the performance of both methods in terms of their MSE as a function of the sparsity factor for $S N R=60 \mathrm{~dB}$, whereas Fig. 10 shows their performance as a function of the SNR for $\eta=0.7$. In both cases the MSE values are obtained averaging 1000 simulations for each SNR and sparsity factor. On the one hand, from Fig. 10 we notice that the RSC performs better for low and high signal to noise ratios (below $25 \mathrm{~dB}$ and above $40 \mathrm{~dB}$ in this case) with an improvement in MSE above $15 \mathrm{~dB}$ in some cases, whereas the histogram seems to provide some advantage in the intermediate region (30 and $35 \mathrm{~dB}$ ). On the other hand, from Fig. 9 we see that the performance of both algorithms depends greatly on the sparsity factor, since the MSE obtained improves notably as $\eta$ increases from

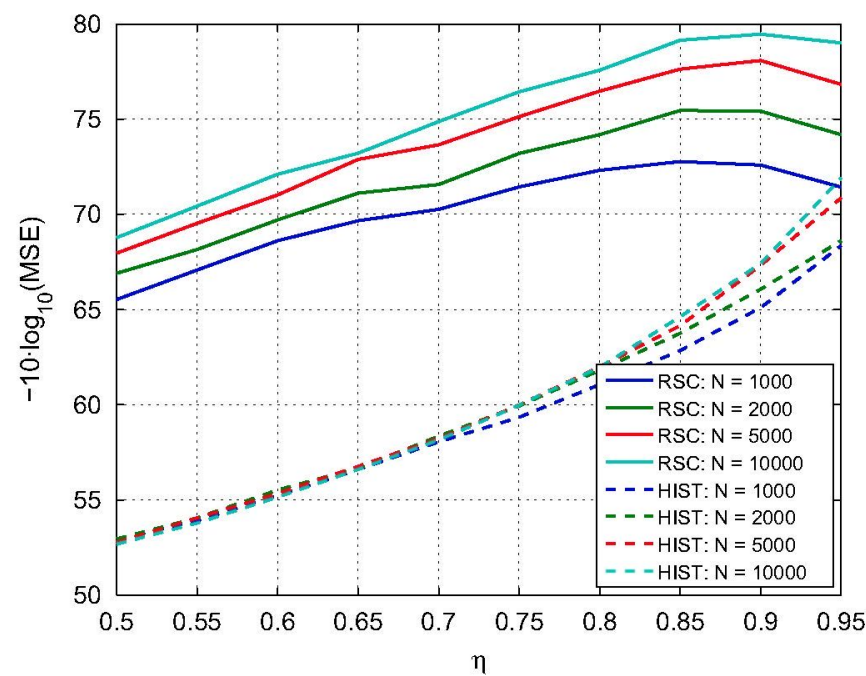

Fig. 9. MSE for the instantaneous noisy case estimating the $2 \times 3$ matrix in (64) with the histogram and the RSC algorithm as a function of $\eta$ and $N$ for $\mathrm{SNR}=60 \mathrm{~dB}$.

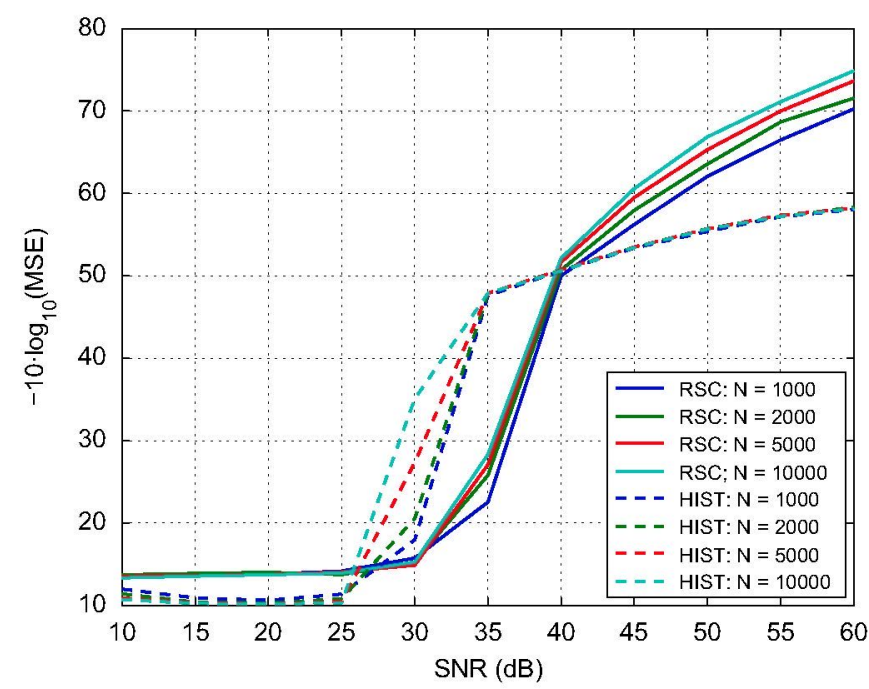

Fig. 10. MSE for the instantaneous noisy case estimating the $2 \times 3$ matrix in (64) with the histogram and the RSC algorithm as a function of the SNR and $N$ for $\eta=0.7$. 
0.5 to 0.8 . For higher sparsity factors the MSE obtained by the RSC algorithm does not improve much more (and even becomes worse for $\eta=0.95$ ), since the number of useful samples decreases (see Fig. 6), whereas the MSE obtained by the histogram keeps improving, as the peaks become more prominent, compensating the decrease in the number of useful samples.

Finally, in order to analyse the behaviour of the RSC and histogram algorithms for different mixing matrices, we have generated 20 random matrices following the method described in Section 6.1, performing 1000 simulations for each value of SNR, $N, \eta$ and model (B-G, B-L and B-U). The resulting MSE is shown in Table 5 as a function of $N$ for two fixed sparsity factors $(\eta=0.7$ and $\eta=0.9$ ), and in Table 6 as a function of the SNR for the same two sparsity factors. On the one hand, the RSC algorithm provides better results than the histogram-based method overall, especially for moderate sparsity factors (e.g. $\eta=0.7$ ) and large SNRs. On the other hand, the histogram-based method seems to provide some marginal advantage for highly sparse signals (e.g. $\eta=0.9$ ), particularly for the B-L and B-U models.

Regarding the three models considered, the best results are always obtained for the B-U model, whereas the B-L model provides the worst results, with the B-G model falling in between. This behaviour can be easily explained calculating the nondetection probability, i.e. the probability of a valid sample falling below the norm threshold, for each model. Integrating (9) between $-A_{\min }$ and $A_{\min }$, we obtain the non-detection probability

Table 5

Average value of $-10 \log _{10}$ (MSE), as a function of $\eta$ and $N$, for noisy instantaneous mixtures with 20 random mixing matrices, $S N R=60 \mathrm{~dB}, Q=2$, and $L=3$.

\begin{tabular}{|c|c|c|c|c|c|c|c|}
\hline \multirow[t]{2}{*}{$\eta$} & \multirow[t]{2}{*}{$N$} & \multicolumn{3}{|l|}{$\mathrm{RSC}$} & \multicolumn{3}{|c|}{ Histogram } \\
\hline & & $B-G$ & B-L & $\mathrm{B}-\mathrm{U}$ & B-G & B-L & B-U \\
\hline \multirow[t]{4}{*}{0.7} & 1000 & 72.3 & 69.1 & 74.5 & 65.9 & 66.4 & 71.3 \\
\hline & 2000 & 74.2 & 70.8 & 76.5 & 66.8 & 67.8 & 72.3 \\
\hline & 5000 & 76.3 & 72.4 & 78.7 & 67.5 & 69.1 & 73.1 \\
\hline & 10000 & 78.1 & 72.9 & 80.6 & 67.8 & 69.6 & 73.4 \\
\hline \multirow[t]{4}{*}{0.9} & 1000 & 73.4 & 70.8 & 75.2 & 72.6 & 71.7 & 78.4 \\
\hline & 2000 & 75.9 & 72.9 & 77.5 & 75.1 & 74.2 & 80.4 \\
\hline & 5000 & 78.7 & 75.4 & 80.7 & 77.1 & 76.8 & 82.4 \\
\hline & 10000 & 80.5 & 76.4 & 82.3 & 78.4 & 78.6 & 83.4 \\
\hline
\end{tabular}

\section{Table 6}

Average value of $-10 \log _{10}$ (MSE), as a function of the SNR, for noisy instantaneous mixtures with 20 random mixing matrices, $N=10000, Q=2$, and $L=3$.

\begin{tabular}{|c|c|c|c|c|c|c|c|}
\hline \multirow[t]{2}{*}{$\eta$} & \multirow[t]{2}{*}{$\mathrm{SNR}(\mathrm{dB})$} & \multicolumn{3}{|l|}{ RSC } & \multicolumn{3}{|c|}{ Histogram } \\
\hline & & $\mathrm{B}-\mathrm{G}$ & B-L & $\mathrm{B}-\mathrm{U}$ & $\mathrm{B}-\mathrm{G}$ & B-L & B-U \\
\hline \multirow[t]{7}{*}{0.7} & 20 & 3.7 & 3.7 & 3.0 & 12.4 & 4.9 & 5.4 \\
\hline & 30 & 7.6 & 7.8 & 6.4 & 46.8 & 48.0 & 46.2 \\
\hline & 40 & 53.4 & 56.1 & 52.9 & 53.5 & 55.2 & 52.5 \\
\hline & 50 & 71.2 & 62.9 & 69.7 & 62.7 & 64.4 & 64.2 \\
\hline & 60 & 78.1 & 72.9 & 80.6 & 67.8 & 69.6 & 73.4 \\
\hline & 70 & 85.3 & 81.4 & 87.5 & 69.3 & 70.8 & 76.0 \\
\hline & 80 & 90.1 & 87.7 & 92.1 & 69.7 & 71.0 & 76.5 \\
\hline \multirow[t]{7}{*}{0.9} & 20 & 5.9 & 6.6 & 4.9 & 20.9 & 14.9 & 15.1 \\
\hline & 30 & 13.9 & 15.0 & 12.2 & 49.7 & 51.2 & 48.2 \\
\hline & 40 & 56.5 & 56.0 & 57.7 & 59.9 & 61.4 & 58.6 \\
\hline & 50 & 69.3 & 69.2 & 73.8 & 71.5 & 72.9 & 72.2 \\
\hline & 60 & 80.5 & 76.4 & 82.3 & 78.4 & 78.6 & 83.4 \\
\hline & 70 & 86.6 & 83.8 & 87.8 & 80.1 & 79.6 & 86.7 \\
\hline & 80 & 90.3 & 88.6 & 90.9 & 80.5 & 79.8 & 87.3 \\
\hline
\end{tabular}

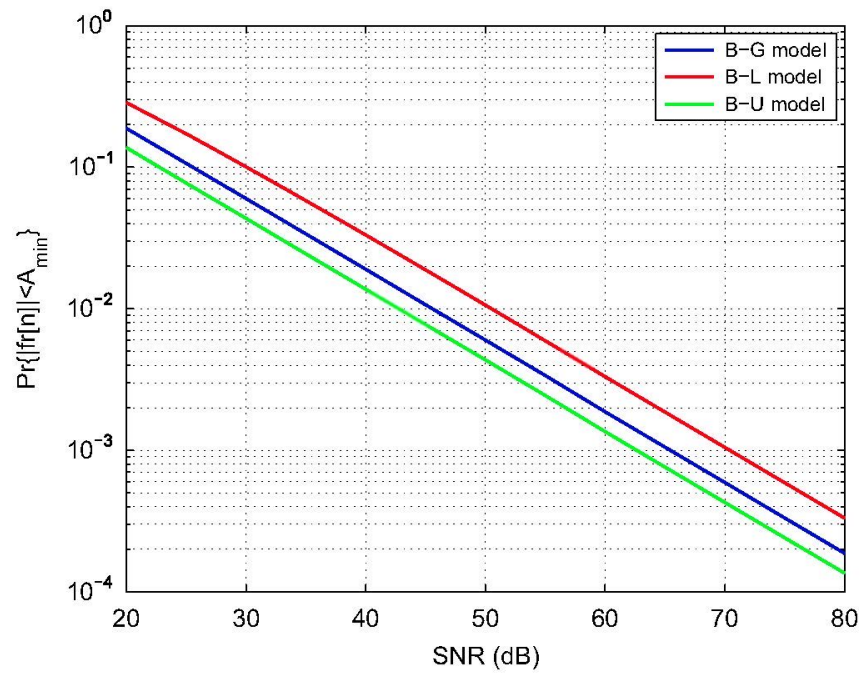

Fig. 11. Non-detection probability, $P_{n d}$, for the B-G, B-L and B-U models as a function of the SNR.

for the B-G model in the noiseless case:

$P_{n d}=\operatorname{Pr}\left\{\left|f_{r}[n]\right|<A_{\min }\right\}=\operatorname{erf}\left(\sqrt{\frac{E_{\min }}{2 \sigma_{f}^{2}}}\right)$,

where erf $(x)$ denotes the well-known error function [44]. Similarly, integrating (11), the non-detection probability for the B-L model becomes

$P_{n d}=\operatorname{Pr}\left\{\left|f_{r}[n]\right|<A_{\min }\right\}=1-\exp \left(-\sqrt{\frac{2 E_{\min }}{\sigma_{f}^{2}}}\right)$,

whereas, integrating (13), for the B-U model we have

$P_{n d}=\operatorname{Pr}\left\{\left|f_{r}[n]\right|<A_{\min }\right\}=\sqrt{\frac{E_{\min }}{3 \sigma_{f}^{2}}}$.

Fig. 11 displays these three probabilities as a function of the SNR, showing that the lowest $P_{n d}$ corresponds to the B-U model, whereas the B-L model presents the highest $P_{n d}$. This implies that the number of useful samples will be typically higher for the B-U model than for the B-G model, which will in turn have a higher number of useful samples than the B-L model, thus leading to better numerical results in the end.

\subsubsection{More than two outputs}

Once more, given the unfeasibility of the histogram-based algorithm for $Q>2$, in this section we simply analyse the performance of the noisy RSC algorithm. We use again the B-G model with $\sigma_{f}^{2}=1$ for the inputs and the same parameters as in Section 6.3.1, except for $S N R_{0}(\mathrm{~dB})$, which is set to 50 , and $N_{b}=30$, thus obtaining $D_{\max }=\pi / 60$ and $\tilde{D}_{\max }=\pi / 20$. $^{5}$

First of all, Fig. 12 shows an example of the MSE for $Q=3$ and a mixing matrix $\mathbf{H}_{2}$ constructed using (65) as a function of the SNR for several values of $N$ and sparsity factors, whereas Fig. 13 does the same for $Q=5$ and $\mathbf{H}_{3}$ obtained from (66). In both cases the behaviour of the RSC algorithm is similar to the $Q=2$ case: its performance improves dramatically above an SNR threshold, which is located around $40 \mathrm{~dB}$ in this case. Increasing the number of samples available or the sparsity factor improves the results,

\footnotetext{
${ }^{5}$ Note that increasing the colinearity thresholds, $D_{\max }$ and $\tilde{D}_{\max }$, allows us to reduce the effect of noise dispersion around the true angles without decreasing the performance of the algorithm, since there are more angular directions available to discriminate between basis vectors.
} 


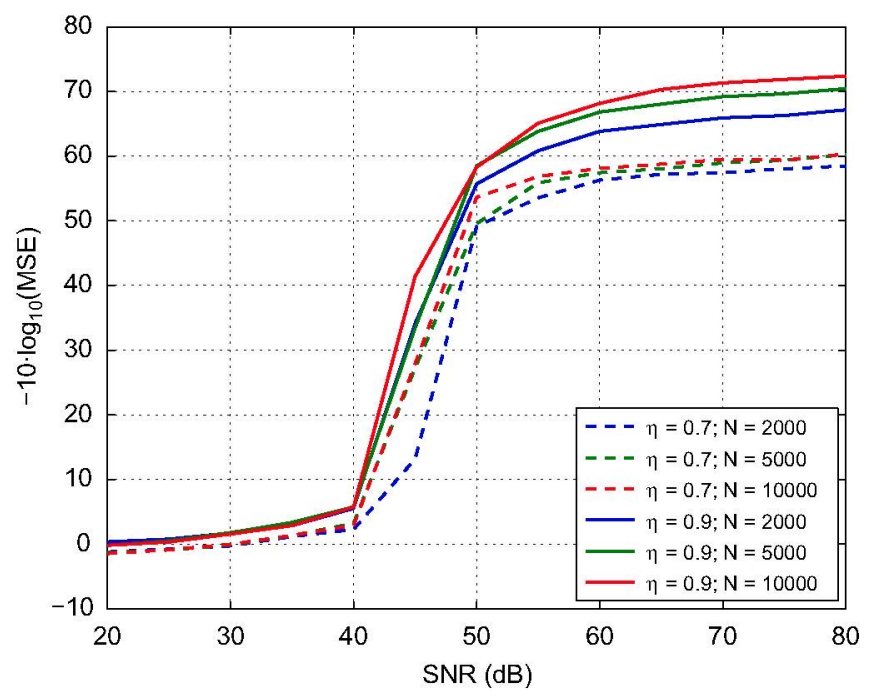

Fig. 12. MSE obtained in the instantaneous noisy case estimating the $3 \times 5$ matrix $\mathbf{H}_{2}$ with the RSC algorithm as a function of the SNR and $N$ for $\eta=0.7$ and $\eta=0.9$.

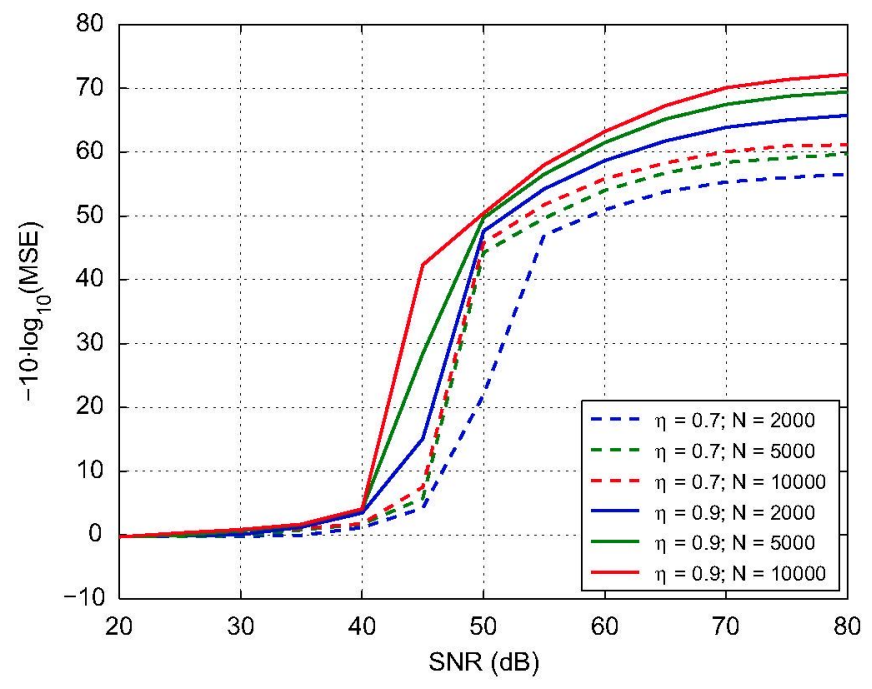

Fig. 13. MSE obtained in the instantaneous noisy case estimating the $5 \times 8$ matrix $\mathbf{H}_{3}$ with the RSC algorithm as a function of the SNR and $N$ for $\eta=0.7$ and $\eta=0.9$.

although the qualitative behaviour of the method does not change.

Finally, we also study the behaviour of the RSC algorithm for different mixing matrices, following the same procedure used in Section 6.3.1: generating 20 random matrices following the method described in Section 6.1 and performing 1000 simulations for each value of SNR, $N, \eta$ and model (B-G, B-L and B-U). The resulting MSE is shown in Table 7 as a function of the SNR for $N=10000$ and $\eta=0.9$. We note that the results are again consistent with the $Q=2$ case: the B-U model is still the best, the B-L is the worst and the B-G model lies somewhere in between. Qualitatively the results are also similar to those shown in Figs. 12 and 13: the performance of the algorithm improves dramatically above a minimum SNR that depends on the case (i.e. the values of $Q$ and $L$ ) and the model.

\subsection{Synthetic data: convolutive case}

The RSC algorithm focuses on the estimation of the mixing matrix, regardless of whether the mixture is instantaneous or convolutive. However, convolutive mixtures may easily result in large mixing matrices, and the number of useful samples for the
Table 7

Average value of $-\mathbf{1 0} \log _{10}$ (MSE), as a function of the SNR, for noisy instantaneous mixtures with 20 randomly generated mixing matrices, $\eta=0.9$ and $N=10000$.

\begin{tabular}{rlrrr}
\hline$(Q L)$ & SNR $(\mathrm{dB})$ & B-G & \multicolumn{1}{c}{ B-L } & B-U \\
\hline$(3,5)$ & 20 & 2.4 & 2.4 & 2.8 \\
& 30 & 4.9 & 4.4 & 6.3 \\
& 40 & 11.7 & 9.7 & 15.1 \\
& 50 & 36.6 & 32.7 & 40.4 \\
& 60 & 68.3 & 44.0 & 67.4 \\
& 70 & 70.6 & 68.9 & 72.3 \\
& 80 & 71.8 & 70.5 & 73.6 \\
$(5,8)$ & 20 & -0.9 & -0.8 & -0.8 \\
& 30 & 0.1 & 0.1 & 0.1 \\
& 40 & 24.9 & 3.1 & 3.1 \\
& 50 & 44.8 & 18.4 & 21.9 \\
& 60 & 67.0 & 60.6 & 63.1 \\
& 70 & 68.0 & 66.1 & 69.0 \\
& 80 & 68.4 & 66.6 & 71.2 \\
\hline
\end{tabular}

algorithm may decrease slightly due to the channel's memory (especially for large values of $L_{r}$ ). Hence, in this section we provide a simulation for a noisy convolutive mixture. The goals of the simulation are two. On the one hand, showing that the performance of the RSC algorithm is similar to the one obtained for an instantaneous mixture. On the other hand, finding out the maximum dimension of the mixing matrix for which the RSC algorithm still provides acceptable results. We perform a simulation using the $B-G$ model with $Q=5$ outputs, $R=2$ sources, sparsity factor $\eta=0.9$, $N=10000$ samples, $\mathrm{SNR}=80 \mathrm{~dB}$, and channels' lengths $L_{q r}=L_{r}=L / 2$, with $L_{r}$ changing from 1 to 15 . We notice that, even though the number of sources is smaller than the number of outputs, the problem becomes under-determined as soon as $L_{r}>2$, since the dimension of the mixing matrix is $Q \times L$, which ranges from $5 \times 2$ for $L_{r}=1$ up to $5 \times 30$ for $L_{r}=15$. For each case 20 mixing matrices are randomly generated following the procedure described in Section 6.1 and 100 simulations are performed.

The results are shown in Fig. 14. For $2 \leq L \leq 14$ the average MSE lies between 64.9 and $76.6 \mathrm{~dB}$, which is similar to the $68.4 \mathrm{~dB}$ obtained in the instantaneous case for $5 \times 8$ mixing matrices (see Table 7). For larger values of $L$ the average MSE falls drastically from $43.0 \mathrm{~dB}$ for $L=16$, down to $20.3 \mathrm{~dB}$ for $L=18$ and $14.6 \mathrm{~dB}$ for $L=30$, implying that some clustering directions are not properly identified. However, we notice that the maximum MSE value falls down much more smoothly, remaining above $50 \mathrm{~dB}$ in all cases. This shows that, although the risk of having ill-conditioned matrices and undetected directions increases with $L$, there are some matrices which can be properly estimated even for large values of $L$. For example, for $L=18$ an MSE above $60 \mathrm{~dB}$ is obtained for 14 out of the 20 matrices, but the poor MSE obtained in the remaining six cases decreases the average MSE down to $20.3 \mathrm{~dB}$. Hence, as a conclusion we remark that the performance of the algorithm depends on the ratio between the number of columns and rows of the mixing matrix (with good results obtained up to $L / Q \approx 3$ ) and not on whether the mixture is instantaneous or convolutive.

\subsection{Real data}

In this section we present some results with real data. We focus on musical and voice signals, making use of two of the data sets introduced in [9] and performing similar experiments to show the potential of our approach. ${ }^{6}$

\footnotetext{
${ }^{6}$ All the data sets used in [9] are freely available to download from http:// www.ac.upc.es/homes/pau/.
} 


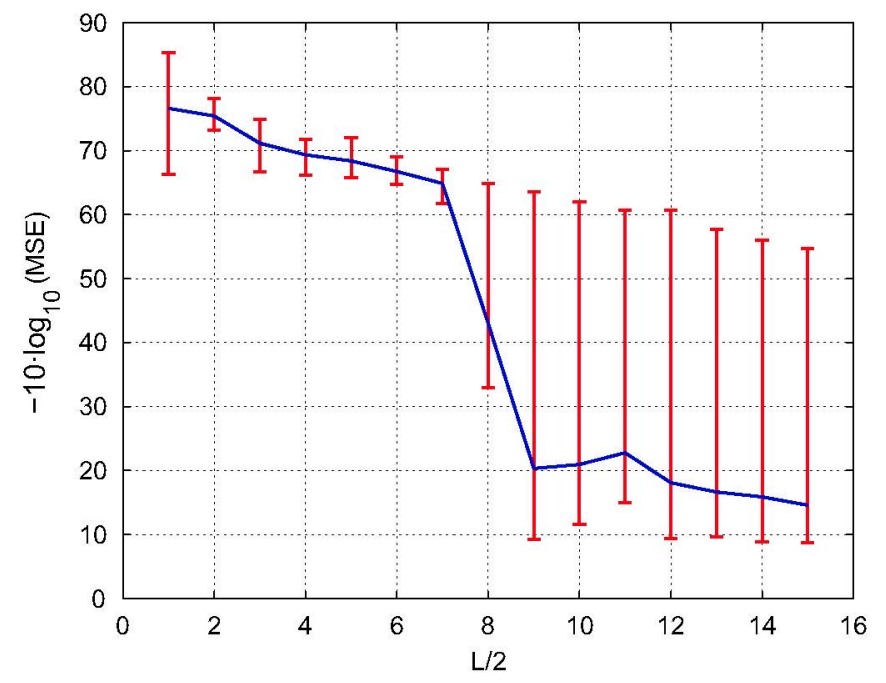

Fig. 14. Average, minimum and maximum MSE for the convolutive noisy case estimating a $5 \times L(2 \leq L \leq 30)$ matrix with the RSC algorithm for $\mathrm{SNR}=80 \mathrm{~dB}$, $\eta=0.9$ and $N=10000$.

\subsubsection{Six flutes experiment}

The first experiment is performed with the SixFlutes data set: the sound of a flute playing six different steady musical notes, digitally recorded with a sampling frequency $f_{s}=44.1 \mathrm{kHz}$ and 16 resolution bits. From this recording, $N=32760$ samples were taken to construct each of the $R=6$ inputs, which were then mixed to obtain the $Q=3$ outputs using $M=1000$ different $3 \times 6$ mixing matrices (i.e. we consider instantaneous mixtures) randomly generated following the procedure described in Section 6.1. Although the resulting mixtures are not sparse at all in the time domain, transforming them into the frequency domain using a single FFT provides us with a sparse output signal where we can apply our algorithm. This situation is clearly illustrated in Fig. 15, which shows the scatter plot both in the time and the frequency domains. On the one hand, in the time domain (Fig. 15(a)) the outputs do not tend to cluster around the directions of the mixing matrix and the RSC algorithm cannot be applied. On the other hand, in the frequency domain (Fig. 15(b)) the clusters around the directions of the mixing matrix can be clearly distinguished and the RSC algorithm (applied with $A_{\min }=20,{ }^{7}$ $D_{\max }=\pi / 60, \tilde{D}_{\max }=\pi / 20$ and $N_{\min }=40$ ) is able to estimate the mixing matrix with an average MSE of $22.6 \mathrm{~dB}$.

\subsubsection{Five songs experiment}

As a second experiment, we consider the FiveSongs data set: five musical pieces (two classical and three pop/folk music) extracted from standard CDs (i.e. $f_{\mathrm{s}}=44.1 \mathrm{KHz}$ and 16 resolution bits), downsampled to $f_{s}=11.025 \mathrm{KHz}$ monophonic signals. Instantaneous mixtures are generated from these signals taking $N=52821$ samples to construct each of the $R=5$ inputs and generating $M=1000$ different $3 \times 5$ mixing matrices randomly according to the procedure described in Section 6.1. Once more, since the signals are not sparse in the time domain, we apply the FFT to represent them in the frequency domain. However, unlike the previous example, these signals are not very sparse in the frequency domain either, as can be seen in the scatter plot shown in Fig. 16(a). Applying the RSC algorithm with $A_{\min }=50,{ }^{8} D_{\max }=\pi / 60, \tilde{D}_{\max }=\pi / 20$ and $N_{\min }=40$ we are often able to extract the true clustering directions imposed

\footnotetext{
${ }^{7}$ The large value of $A_{\min }$ is due to the fact that we do not require the output signals to be normalized, so $\max \left|Y_{q}(\omega)\right| \approx 4545.4$.

${ }^{8}$ The large value of $A_{\min }$ is again due to the fact that the output signals are not normalized, so we have $\max \left|Y_{q}(\omega)\right| \approx 494.4$.
}
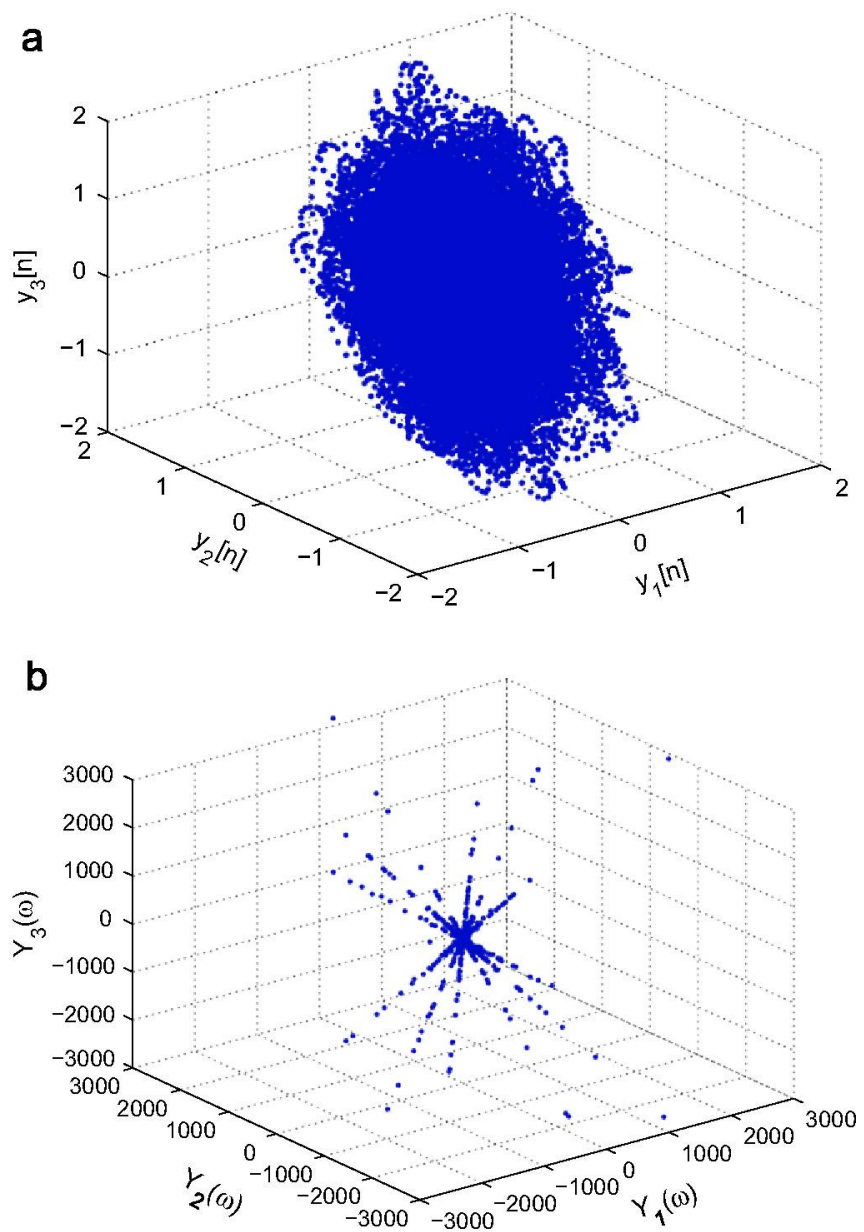

Fig. 15. Example of a three-dimensional scatter plot for the six flutes experiment ( $Q=3$ outputs, $L=6$ inputs). (a) Scatter plot in the time domain. (b) Scatter plot in the frequency domain.

by the columns of the mixing matrix with an MSE up to $25.8 \mathrm{~dB}$, as shown in Fig. 16(b), and recover the original sources quite reliably using the pseudoinverse of the estimated matrix, as shown in Fig. 17. Unfortunately, for some ill-conditioned mixing matrices one or more clustering directions are not properly estimated, so the average value of the MSE is only $4.90 \mathrm{~dB}$. Nevertheless, if we consider only the four best clustering directions this MSE becomes $11.43 \mathrm{~dB}$ and for the best three directions we obtain $18.56 \mathrm{~dB}$, which is close to the value obtained for the previous experiment, where we always achieve a good estimation for all the clustering directions.

As a conclusion, we remark that this data set is not sparse enough for the application of our algorithm even in the frequency domain. Hence, we are always able to properly estimate the best three clustering directions, but the fourth and fifth directions are sometimes not properly estimated.

\section{Conclusions and future lines}

In this paper we have proposed a novel random search clustering (RSC) algorithm for mixing matrix identification in MIMO linear blind inverse problems with sparse inputs. The method exploits the sparsity of the inputs to estimate the columns of the mixing matrix, which can be seen as the basis vectors in a non-orthogonal expansion, by randomly selecting an observation and accepting or discarding it based on the cardinality of its colinearity set. The proposed approach is able to work with any number of inputs and 

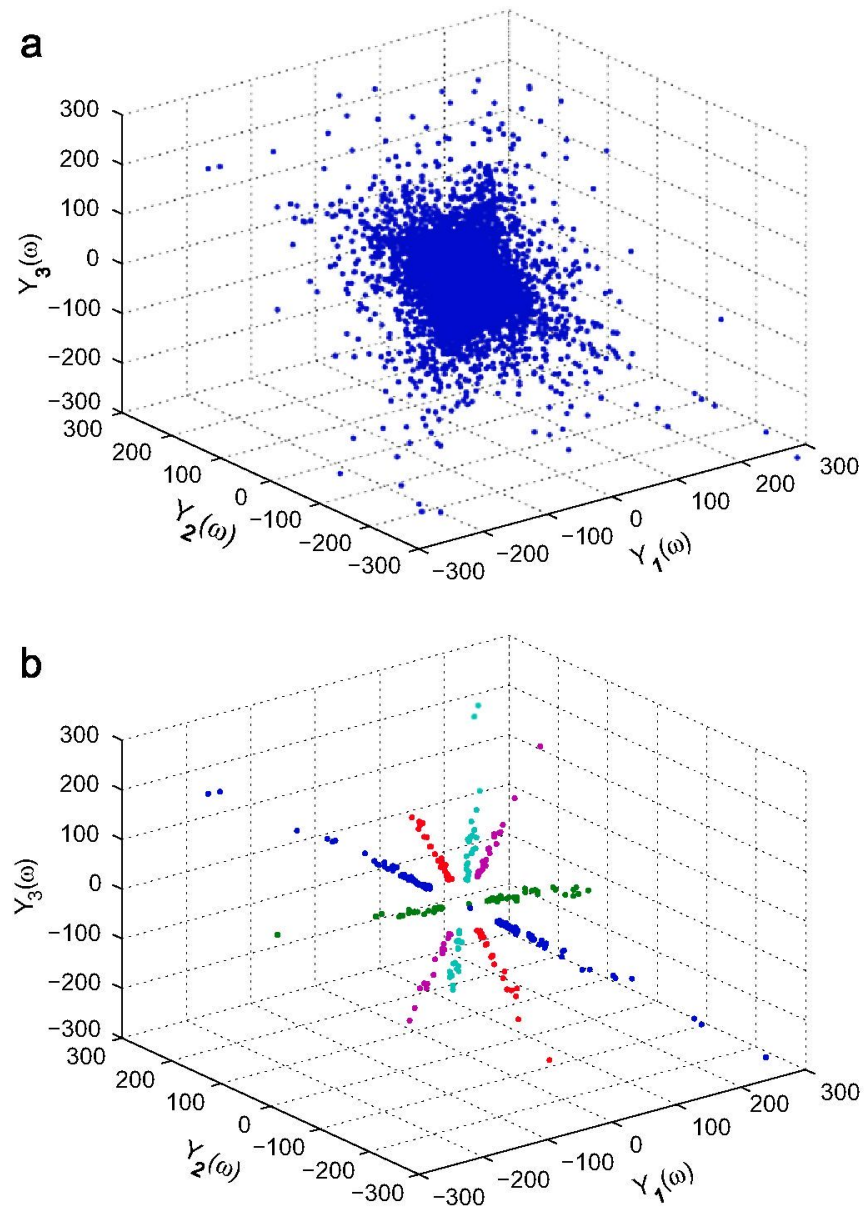

Fig. 16. Example of a three-dimensional scatter plot for the five songs experiment $(Q=3$ outputs, $L=5$ inputs) in the frequency domain. (a) Full scatter plot. (b) Scatter plot of the recovered sources $\left(-10 \log _{10}(\mathrm{MSE})=25.8 \mathrm{~dB}\right)$.
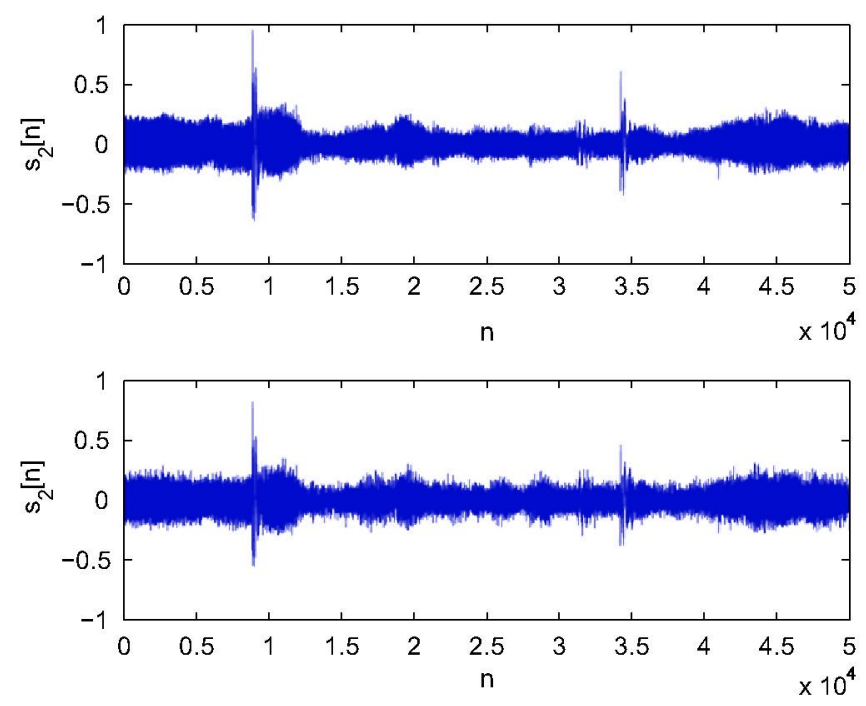

Fig. 17. Example of a true input signal (top) and the signal recovered using the pseudoinverse of the estimated matrix (bottom) for the five songs experiment.

outputs, instantaneous and convolutive mixtures, and its computational cost grows linearly with the number of samples, sources and observations. Moreover, besides their sparsity no specific PDF for the inputs has been assumed, thus making the method applicable to a wide range of problems. Simulation results with synthetic data using the Bernoulli-Gaussian, Bernoulli-Laplacian and BernoulliUniform models confirm its excellent performance for moderate/ high SNRs, with a behaviour not depending on the output dimension, but rather on the number of inputs/outputs ratio. Finally, two examples with real data (musical notes and songs) have been provided, showing the good performance of the algorithm in the transformed Fourier domain.

In this paper we have concentrated on the development of the algorithm and its theoretical aspects, including a robust and general parameter selection procedure. Hence, a first future line of research is analysing its performance more thoroughly with real data, such as biomedical signals (e.g. electrograms recorded on patients with atrial fibrillation) or speech/audio signals. Additional lines of research include extending the proposed approach for exploiting any observation where no more than $Q$ inputs are active, developing an on-line algorithm for detecting the number of inputs and estimating them simultaneously, or even applying it to post-nonlinear mixtures.

\section{Acknowledgement}

We would like to thank the reviewers for their many comments and suggestions which helped to improve substantially the quality of the paper.

\section{Appendix A. Angular error for the noisy case}

For the noisy case, the first component of the angular vector, $\varphi\left(\mathbf{y}_{\ell}[n]\right)$, when only the $\ell$-th source is active is given by

$\varphi_{1}\left(\mathbf{y}_{\ell}[n]\right)=\arctan \left(\frac{y_{\ell}^{(2)}[n]}{y_{\ell}^{(1)}[n]}\right)=\arctan \left(\frac{x_{\ell}^{(2)}[n]}{x_{\ell}^{(1)}[n]}+\xi_{\ell}^{(1)}[n]\right)$,

where we have used Taylor's theorem for $(1+x)^{-1}$ around $x=0$ with a single term, and

$\xi_{\ell}^{(1)}[n]=\frac{w_{2}[n]}{x_{\ell}^{(1)}[n]}-\frac{w_{1}[n] y_{\ell}^{(2)}[n]}{\left(x_{\ell}^{(1)}[n]\left(1+\varepsilon_{\ell}^{(1)}[n]\right)\right)^{2}}$

is the Lagrange form of the remainder, with $0 \leq\left|\varepsilon_{\ell}^{(1)}[n]\right| \leq$ $\left|w_{1}[n] / x_{\ell}^{(1)}[n]\right|$. Applying again Taylor's theorem to $\arctan (x)$ around $x=x_{\ell}^{(2)}[n] / x_{\ell}^{(1)}[n]$ using a single term, $\varphi_{1}\left(\mathbf{y}_{\ell}[n]\right)=\tilde{\theta}_{1, \ell}+\Delta \theta_{1, \ell}$, with

$\Delta \theta_{1, \ell}=\frac{\xi_{\ell}^{(1)}[n]}{1+\left(\delta_{\ell}^{(1)}[n]\right)^{2}}$,

and $\left|x_{\ell}^{(2)}[n] / x_{\ell}^{(1)}[n]\right|-\left|\xi_{\ell}^{(1)}[n]\right| \leq\left|\delta_{\ell}^{(1)}[n]\right| \leq\left|x_{\ell}^{(2)}[n] / x_{\ell}^{(1)}[n]\right|+\left|\xi_{\ell}^{(1)}[n]\right|$ for the remainder.

Following a similar procedure for $2 \leq q \leq Q-1$ we obtain $\varphi_{q}\left(\mathbf{y}_{\ell}[n]\right)=\operatorname{sign}\left(y_{\ell}^{(1)}[n]\right) s_{\ell}[n] \theta_{q, \ell}+\Delta \theta_{q, \ell}$, with

$\Delta \theta_{q, \ell}=\frac{\xi_{\ell}^{(q)}[n]}{1+\left(\delta_{\ell}^{(q)}[n]\right)^{2}}$,

where $\operatorname{sign}\left(y_{\ell}^{(1)}[n]\right) s_{\ell}[n]=1$ for $x_{\ell}^{(1)}[n] \geq w_{1}[n]$,

$\xi_{\ell}^{(q)}[n]=\frac{w_{q+1}[n]}{\left\|\mathbf{x}_{\ell}^{(1: q)}[n]\right\|}-\frac{y_{\ell}^{(q+1)}[n]}{\left\|\mathbf{x}_{\ell}^{(1: q)}[n]\right\|} \times \frac{2 \mathbf{x}_{\ell}^{(1: q)}[n]^{\top} \mathbf{w}_{1: q}[n]+\left\|\mathbf{w}_{1: q}[n]\right\|^{2}}{2\left\|\mathbf{x}_{\ell}^{(1: q)}[n]\right\|^{2}\left(1+\varepsilon_{\ell}^{(q)}[n]\right)^{3 / 2}}$

the first remainder is given by

$0 \leq\left|\varepsilon_{\ell}^{(q)}[n]\right| \leq\left(2\left|\mathbf{x}_{\ell}^{(1: q)}[n]^{\top} \mathbf{w}_{1: q}[n]\right|+\left\|\mathbf{w}_{1: q}[n]\right\|^{2}\right) /\left\|\mathbf{x}_{\ell}^{(1: q)}[n]\right\|^{2}$,

and the second remainder by

$$
\begin{aligned}
& \left|x_{\ell}^{(q+1)}[n]\right| / \|\left.\mathbf{x}_{\ell}^{(1: q)}[n]\right|^{2}-\left|\xi_{\ell}^{(q)}[n]\right| \leq\left|\delta_{\ell}^{(q)}[n]\right| \\
& \quad \leq\left|x_{\ell}^{(q+1)}[n]\right| /\left\|\mathbf{x}_{\ell}^{(1: q)}[n]\right\|^{2}+\left|\xi_{\ell}^{(q)}[n]\right| .
\end{aligned}
$$




\section{References}

[1] A. Hyvärinen, J. Karhunen, E. Oja, Independent Component Analysis, John Wiley \& Sons, New York, NY, USA, 2001.

[2] A. Cichocki, S. Amari, Adaptive Blind Signal and Image Processing, John Wiley \& Sons, Chichester, UK, 2002

[3] P. Comon, C. Jutten, Handbook of Blind Source Separation: Independent Component Analysis and Applications, Elsevier, Oxford, UK, 2010.

[4] J.M. Mendel, C.S. Burrus, Maximum-Likelihood Deconvolution: A Journey into Model-Based Signal Processing, Springer, 1989.

[5] P. Campisi, K. Egiazarian (Eds.), Blind Image Deconvolution: Theory and Applications, CRC Press, Boca Raton, FL, USA, 2007.

[6] Z. Ding, Y. Li, Blind Equalization and Identification, Marcel Dekker, New York, NY, USA, 2001

[7] C.-Y. Chi, C.-C. Feng, C.-H. Chen, C.-Y. Chen, Blind Equalization and System Identification: Batch Processing Algorithms, Performance and Applications, Springer-Verlag, London, UK, 2006.

[8] T.-W. Lee, M.S. Lewicki, M. Girolami, T.J. Sejnowski, Blind source separation of more sources than mixtures using overcomplete representations, IEEE Signal Process. Lett. 6 (4) (1999) 87-90.

[9] P. Bofill, M. Zibulevsky, Underdetermined blind source separation using sparse representations, Signal Process. 81 (11) (2001) 2353-2362.

[10] D. Erdogmus, L. Vielva, J.C. Príncipe, Nonparametric estimation and tracking of the mixing matrix for underdetermined blind source separation, in: Proceedings of the 2 nd International Symposium on Independent Component Analysis and Blind Signal Separation (ICA), 2001, pp. 189-193.

[11] D. Luengo, I. Santamaría, J. Ibáñez, L. Vielva, C. Pantaleón, A fast blind SIMo channel identification algorithm for sparse sources, IEEE Signal Process. Lett. 10 (5) (2003) 148-151.

[12] P. Kisilev, M. Zibulevsky, Y.Y. Zeevi, A multiscale framework for blind separation of linearly mixed signals, J. Mach. Learn. Res. (2003) 1339-1364.

[13] F.J. Theis, E.W. Lang, C.G. Puntonet, A geometric algorithm for overcomplete linear ICA, Neurocomputing 56 (2004) 381-396.

[14] P.D. O'Grady, B.A. Pearlmutter, Hard-LOST: modified $k$-means for oriented lines, in: Proceedings of the Irish Signals and Systems Conference (ISSC), Belfast, UK, 2004, pp. 247-252.

[15] Y.Q. Li, A. Cichocki, S.-I. Amari, Analysis of sparse representation and blind source separation, Neural Comput. 16 (2006) 1193-1234.

[16] R. Mahdian, M. Babaiezadeh, C. Jutten, Separation of speech sources in underdetermined case using SCA and time-frequency methods, in: Proceedings of the International Symposium on Telecommunications, 2008, pp. 533-538.

[17] P.D. O'Grady, B.A. Pearlmutter, The LOST algorithm: finding lines and separating speech mixtures, EURASIP J. Adv. Signal Process. 23 (2008). $17 \mathrm{pp}$.

[18] M. Aharon, M. Elad, A.M. Bruckstein, K-SVD and its non-negative variant for dictionary design, in: Proceedings of the SPIE Conference on Wavelets, 2005 , pp. 90-97.

[19] Z. He, A. Cichocki, K-EVD clustering and its application to sparse component analysis, in: Proceedings of the 7th International Symposium on Independent Component Analysis and Blind Signal Separation (ICA), 2006, pp. 90-97.

[20] S. Van Vaerenbergh, I. Santamaria, A spectral clustering approach to underdetermined postnonlinear blind source separation of sparse sources, IEEE Trans. Neural Networks 17 (3) (2006) 811-814.

[21] L. Vielva, Y. Pereiro, D. Erdogmus, J.C. Príncipe, Inversion techniques for underdetermined BSS in an arbitrary number of dimensions, in: Proceedings of the 4th International Symposium on Independent Component Analysis and Blind Signal Separation (ICA), Nara, Japan, 2003, pp. 131-136.

[22] S.M. Kay, Fundamentals of Statistical Signal Processing. Volume 2: Detection TheoryPrentice-Hall, Upper Saddle River, NJ, USA, 2002.

[23] D. Luengo, I. Santamaría, L. Vielva, A general solution to blind inverse problems for sparse input signals, Neurocomputing 69 (1-3) (2005) 198-215.

[24] G.H. Golub, C.F. Van Loan, Matrix Computations, 3rd ed., The John Hopkins University Press, Baltimore, MA, USA, 1996.

[25] M.S. Lewicki, T.J. Sejnowski, Learning overcomplete representations, Neural Comput. 12 (2000) 337-365.

[26] J.M. Mendel, Optimal Seismic Deconvolution: An Estimation Based Approach, Academic Press, 1983.

[27] O. Rosec, J.-M. Boucher, B. Nsiri, T. Chonavel, Blind marine seismic deconvolution using statistical MCMC methods, IEEE J. Oceanic Eng. 28 (3) (2003) 502-512.

[28] D. Ge, J. Idier, E. Le Carpentier, A new MCMC algorithm for blind BernoulliGaussian deconvolution, in: Proceedings of the 16th European Signal Processing Conference (EUSIPCO), Lausanne, Switzerland, 2008.

[29] K.F. Kaaresen, E.B. lviken, Blind deconvolution of ultrasonic traces accounting for pulse variance, IEEE Trans. Ultrason. Ferroelectrics Freq. Control 46 (3) (1999) 564-573.

[30] G. Kail, C. Novak, B. Hofer, F. Hlawatsch, A blind Monte Carlo detectionestimation method for optical coherence tomography, in: Proceedings of the 34th IEEE International Conference on Acoustics, Speech and Signal Processing (ICASSP 2009), Taipei, Taiwan, 2009, pp. 493-496.

[31] O. Rabaste, T. Chonavel, Estimation of multipath channels with long impulse response at low SNR via an MCMC method, IEEE Trans. Signal Process. 55 (4) (2007) 1312-1325.

[32] M. Ghosh, Analysis of the effect of impulse noise on multicarrier and single carrier QAM systems, IEEE Trans. Commun. 44 (2) (1996) 145-147.

[33] F. Abdelkefi, P. Duhamel, F. Alberge, Impulsive noise cancellation in multicarrier transmission, IEEE Trans. Commun. 53 (1) (2005) 94-106.
[34] S. Bourguignon, H. Carfantan, Bernoulli-Gaussian spectral analysis of unevenly spaced astrophysical data, in: Proceedings of the 13th Statistical Signal Processing Workshop (SSP), Bordeaux, France, 2005, pp. 811-816.

[35] S. Gazor, W. Zhang, Speech probability distribution, IEEE Signal Process. Lett. 10 (7) (2003) 204-207

[36] J.-H. Chang, N.S. Kim, Voice activity detection based on multiple statistical models, IEEE Trans. Signal Process. 54 (6) (2006) 1965-1976.

[37] R. Martin, Speech enhancement based on minimum mean-square error estimation and supergaussian priors, IEEE Trans. Speech Audio Process. 13 (5) (2005) 845-856.

[38] S. Alliney, S.A. Ruzinsky, An algorithm for the minimization of mixed $\ell_{1}$ and $\ell_{2}$ norms with application to Bayesian estimation, IEEE Trans. Signal Process. 42 (3) (1994) 618-627.

[39] R. Tibshirani, Regression shrinkage and selection via the Lasso, J. R. Stat. Soc B 58 (1) (1996) 267-288.

[40] M. Ting, R. Raich, A.O. Hero III, Sparse image reconstruction for molecular imaging, IEEE Trans. Image Process. 18 (6) (2009) 1215-1227.

[41] I. Johnstone, B.W. Silverman, Needles and straw in haystacks: empirical Bayes estimates of possibly sparse sequences, Ann. Stat. 32 (4) (2004) 1594-1649.

[42] A. Javanmard, P. Pad, M. Babaie-Zadeh, C. Jutten, Estimating the mixing matrix in underdetermined sparse component analysis (SCA) using consecutive independent component analysis (ICA), in: Proceedings of the 16th European Signal Processing Conference (EUSIPCO), Lausanne, Switzerland, 2008.

[43] H. Stark, J.W. Woods, Probability and Random Processes with Applications to Signal Processing, 3rd ed., Prentice-Hall, Upper Saddle River, NJ, USA, 2002.

[44] M. Abramowitz, l.A. Stegun (Eds.), Handbook of Mathematical Functions: With Formulas, Graphs, and Mathematical Tables, Dover, New York, NY, USA, 1965.

[45] I.S. Gradshteyn, I.M. Ryzhik, Table of Integrals, Series, and Products, 7th ed. Academic Press, Burlington, MA, USA, 2007.

[46] C.M. Bishop, Neural Networks for Pattern Recognition, Clarendon Press, Oxford, UK, 1995.

[47] S. Fiori, Blind adaptation of stable discrete-time IIR filters in state-space form IEEE Transactions on Signal Processing 54 (7) (2006) 2596-2605.

[48] S. Fiori, P. Baldassarri, Approximate joint matrix diagonalization by Riemannian-gradient-based optimization over the unitary group (with application to neural multichannel blind deconvolution), in: E. Chabot, H. D'Arras (Eds.), Neural Computation and Particle Accelerators: Research Technology and Applications, NOVA Pub. 2009. 